Harris A. W., Boslough M., Chapman C. R., Drube L., Michel P., and Harris A. W. (2015) Asteroid impacts and modern civilization: Can we prevent a catastrophe? In Asteroids $I V$ (P. Michel et al., eds.), pp. 835-854. Univ. of Arizona, Tucson, DOI: 10.2458/azu_uapress_9780816532131-ch042.

\title{
Asteroid Impacts and Modern Civilization: Can We Prevent a Catastrophe?
}

\author{
Alan W. Harris \\ German Aerospace Center (DLR) Institute of Planetary Research \\ Mark Boslough \\ Sandia National Laboratories \\ Clark R. Chapman \\ Southwest Research Institute \\ Line Drube \\ German Aerospace Center (DLR) Institute of Planetary Research \\ Patrick Michel \\ Lagrange Laboratory, Université Côte d'Azur, Observatoire de la Côte d'Azur, CNRS
}

\author{
Alan W. Harris \\ MoreData! Inc.
}

\begin{abstract}
We are now approaching the level of technical expertise necessary to deflect a near-Earth asteroid (NEA) capable of destroying a large urban area, if not a small country. The current level of activity in the field, including search programs, physical characterization, and international initiatives to assess mitigation strategies, is unprecedented. However, we have only just started to explore the relevant properties of the small end of the NEA population (diameter $<300 \mathrm{~m}$ ), of which the next major impactor is most likely to be a member. The Chelyabinsk event has reminded us that objects as small as $20 \mathrm{~m}$, about which we know very little, impact frequently and are a serious threat to life and property. Political awareness and international response efforts are still at a very primitive stage. For a global guarantee of protection, advances in scientific and technical competence must be matched by improvements in international coordination, as well as preparedness at the political level.
\end{abstract}

\section{INTRODUCTION}

The planets of the solar system started out as clumps of material, so-called planetesimals, with diameters of around $1-100 \mathrm{~km}$, which grew by accreting particles and small bodies from the cloud of gas and dust that surrounded the newly formed Sun. Some planetesimals enjoyed rapid growth and quickly developed significant gravitational fields, thereby attracting and absorbing more and more small bodies to become fully fledged planets. Others were destroyed in violent collisions that produced clouds of fragments. A large fraction of the asteroids present in the solar system today are collisional fragments, orbiting the Sun in the main asteroid belt between the orbits of Mars and Jupiter. As a result of gradual orbital evolution due to the recoil effect of thermal radiation emission (the Yarkovsky effect; see the chapter by Vokrouhlicky et al. in this volume) and perturbations by large planets, primarily Jupiter, there is a continual leakage of asteroids from the main belt into the inner solar system. The resulting populations of so-called Mars crossers and near-Earth asteroids (NEAs) have orbits that give rise to occasional collisions with the terrestrial planets. The orbits of periodic comets can also evolve to cross Earth's orbit. The population of near-Earth objects (NEOs, a term embracing both asteroids and comets) contains a wide variety of bodies with diverse physical and dynamical properties (see the chapter by Jedicke et al. in this volume), and presents a permanent threat to our civilization and a serious challenge to those involved in developing mitigation strategies. While the term "NEO" is often used in texts on the impact hazard, comets are much less numerous than asteroids in Earth's neighborhood and represent only a minor fraction of potential 
impactors. In this chapter we use the term "NEA" when referring primarily to the asteroid component of the NEO population (e.g., in discussions of population statistics and deflection techniques).

Awareness of the threat presented by NEOs has grown rapidly during the past few decades as a result of, for example, the first observation in real time of a large-scale impact on a planet, i.e., the impact of Comet Shoemaker-Levy 9 on Jupiter in 1994 (e.g., see Noll et al., 1996); observations of fresh craters appearing on the Moon and Mars (e.g., Buratti and Johnson, 2003; Malin et al., 2006); the discovery, with search programs of ever-increasing sophistication, of thousands of NEOs, some of which make uncomfortably close approaches to Earth; and the realization that the Tunguska event of 1908 was not a freak phenomenon after the blast waves from the Chelyabinsk superbolide of February 15, 2013, injured some 1500 people and damaged thousands of buildings (Popova et al., 2013) (see section 2). The rather unsettling coincidence of the anticipated close approach at an altitude of only $28,000 \mathrm{~km}$ on that very same day of (367943) Duende (known at the time by its provisional designation of $2012 \mathrm{DA}_{14}$ ), an asteroid with an extent of about $20 \times 40 \mathrm{~m}$ unrelated to the Chelyabinsk object, ensured that asteroids made the headlines in most major news media at the time.

While this chapter concentrates on developments since the publication of Asteroids III, readers interested in earlier developments in this field are referred to, e.g., Chapman and Morrison (1994), Toon et al. (1997), and the books edited by Gehrels (1994), Belton et al. (2004), and Bobrowsky and Rickman (2007). In nearly all aspects of the impact hazard there have been major advances since the publication of the predecessor chapter to this one (Morrison et al., 2002) in Asteroids III. In terms of our knowledge of the threat, the number of NEAs of all sizes discovered has risen from around 2000 at the end of 2002 to some 11,000 at the time of this writing (section 3). Surveys carried out by spacebased infrared telescopes, such as the Wide-Field Infrared Survey Explorer (WISE) (Wright et al., 2010) and the Spitzer Space Telescope (Werner et al., 2004), have provided diameters and albedos for many hundreds of NEAs, in contrast to the few tens of NEAs for which this information was available in 2002 (see section 5 and the chapter by Mainzer et al. in this volume). There has been a significant increase in efforts by observers and modelers to physically characterize the NEA population in terms of spin vectors, taxonomy, thermal properties, binarity, shape, surface structure, etc., and investigate properties particularly relevant to deflection considerations, such as porosity and internal structure, although these efforts have not kept pace with the dramatic increase in the discovery rate. Three NEAs have been visited by spacecraft, revealing very different small worlds, despite similarities in taxonomic type. The NASA Near Earth Asteroid Rendezvous (NEAR)Shoemaker spacecraft visited the second largest NEA, (433) Eros, for one year starting in February 2000, revealing a peanut-shaped body with rich geological features such as large craters but a lack of small ones, boulders, "ponds," and a fine regolith-covered surface (Cheng, 2002). The Japan
Aerospace Exploration Agency's (JAXA) Hayabusa spacecraft visited the small NEA (25143) Itokawa and returned a surface sample to Earth (Nakamura et al., 2011). Images of Itokawa suggest that even a body of just a few hundred meters in size can have a rubble-pile structure and be covered with large boulders and coarse regolith (Fujiwara et al., 2006; see also the chapter by Yoshikawa et al. in this volume). The Chinese Chang'e-2 lunar mission flew by (4179) Toutatis just $800 \mathrm{~m}$ above its surface when Toutatis came within 18 lunar distances of Earth in December 2012. The images obtained show a contact binary NEA of $4.75 \times 1.95 \mathrm{~km}$ and details of the distribution of boulders and regolith (Huang et al., 2013). Radar observations have provided information on the shapes of some NEAs passing close to Earth (see the chapter by Benner et al. in this volume), including small binary NEAs, which constitute about $15 \%$ of the NEA population.

The Chelyabinsk event (Fig. 1 and section 2) taught us that an Earth-bound object large enough to cause considerable destruction of property, if not life, can be small enough to escape detection, especially if its final approach is in the daylit sky. While new wide-field, rapid-scan telescopes are currently planned to detect small impactors, the days to weeks of warning time they might provide would be inadequate for any mitigation action, apart from advising the relevant communities, who might organize evacuation if considered appropriate and feasible. The size of the threatening NEO that will trigger the first spaceborne deflection attempt is a matter of debate. Due to the steep size distribution of NEOs there are many more small objects than large ones, and consequently many more sightings of fireballs than craterforming impacts. However, in this respect it is informative to compare the airburst of a stony object, such as the Tunguska event, which flattened $2000 \mathrm{~km}^{2}$ of forest but left no other obvious trace on the ground, with the impact of a similarly sized metallic object, which produced the $1.2-\mathrm{km}$-diameter Barringer Crater in Arizona. In both cases the diameter of the impactor is estimated to be $30-50 \mathrm{~m}$. Clearly the potential of a threatening NEO to cause damage depends not only

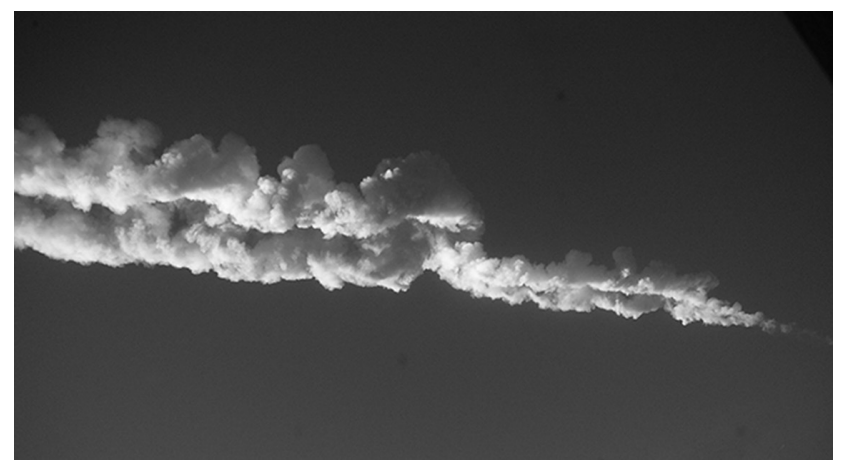

Fig. 1. The trail left by the Chelyabinsk bolide. The left part of the image shows two contrarotating vortices formed by heating and buoyancy effects in the horizontal cylinder of air in which kinetic energy of the asteroid was deposited. Credit: Wikimedia Commons. 
on its size but also on its composition, density, and ability to survive passage through the atmosphere. The asteroid $2008 \mathrm{TC}_{3}$ lost $99.9 \%$ of its mass during its passage through the atmosphere, which suggests that this meter-sized body probably consisted of fragile material, as well as more solid but weakly aggregated pieces. Nevertheless, a few hundred fragments with a surprising range of lithologies were recovered in Sudan (see, for example, the chapters by DeMeo et al. and Borovička et al. in this volume). It seems that the lower size threshold for considering spaceborne deflection action should be around 30-50 m (see the discussion in section 6), although with current and foreseeable search programs it is highly unlikely that an object of this size would be detected far enough in advance for any deflection action to be feasible. At the other end of the size scale, it would seem difficult to justify spending taxpayers' money at present investigating how to deal with an object of more than $300 \mathrm{~m}$ in diameter, given that the impact probability per year of such objects is estimated to be around one in 70,000. In any case, given the size-frequency distribution of NEOs, it is probable that we will gain some experience in deflecting smaller ones before being confronted by a large potential impactor.

Serious effort is now being spent on investigating realistic asteroid deflection techniques (section 4). The main contenders at present are impulsive techniques, such as kinetic and explosive methods, and so-called "slow-push" techniques, such as those based on laser or ion-beam devices. The gravity tractor is a viable contender as a "slow-pull" technique. An interesting concept is the use of a slow technique directly after an impulsive deflection has taken place, in order to make fine corrections to the new orbit of the deflected NEA and provide some insurance against malfunction of the impulse transfer.

In recent years not only have the scientific and spacetechnology communities begun to pay more attention to impact-hazard issues, but some national space agencies and international organizations are now investing significant funding in various endeavors, motivated by the increased awareness of the problem.

NASA now funds the International Astronomical Union's Minor Planet Center and has recently significantly increased its funding of NEO search programs such as Lincoln Near Earth Asteroid Research (LINEAR), the Catalina Sky Survey, and the Panoramic Survey Telescope and Rapid Response System (Pan-STARRS) (see the chapter by Jedicke et al. in this volume). NASA also funds several groups to carry out research into aspects of NEA deflection options, such as those at Iowa State University's Asteroid Deflection Research Center. In total NASA allocated about 3\% of its 2013 NEO funding to mitigation studies. The European Space Agency (ESA) is currently developing a program devoted to NEO defense in the framework of its Space Situational Awareness (SSA) initiative, including establishing a European NEO Coordination Centre. A collaborative mission concept currently under study in the U.S. and Europe is the Asteroid Impact and Deflection Assessment (AIDA) mission, the goal of which is to demonstrate the kinetic impactor concept by impacting the moon of a binary NEA (see section 4).
The European Commission's Seventh Framework Programme for Research and Technological Development (FP7) issued a call for proposals in 2010 entitled "Prevention of impacts from near-Earth objects on our planet." A consortium of 13 partners from 6 countries, including Russia and the U.S., responded with a proposal for the project NEOShield (http://www.neoshield.net), which succeeded in gaining 5.8 million euros of funding from January 2012 to May 2015. The NEOshield partners are investigating mitigation-relevant NEO physical properties and potential mitigation techniques (Harris et al., 2013). A further call for proposals for similar research projects was issued by the Commission in 2013; funding for a second phase of NEOShield, until 2017, was granted while this chapter was in preparation.

In a complementary initiative to those mentioned above, the United Nations (UN) has authorized the establishment of two new bodies (see section 7), the International Asteroid Warning Network (IAWN) and the Space Mission Planning Advisory Group (SMPAG).

While it seems the stage is now set for coordinated international activities in pursuit of solutions to the NEO impact threat, it remains to be seen if the momentum built up over the past few years can be maintained. The activities to be overseen by the IAWN and SMPAG will require significant and continuous funding, which will have to be made available by the participating countries. The recent Chelyabinsk event has served to focus attention on the impact hazard, but it is a fact of life that political interest in an area of public concern tends to wane if not regularly stimulated. Furthermore, we are still far removed from having an international agreement on who would do what, who would pay for what, and who would take responsibility for failure, in the event of a deflection action becoming necessary.

\section{THE CHELYABINSK EXPERIENCE}

On February 15, 2013, at the crack of dawn, a glowing object appeared in the clear southeastern sky seen from the industrial Russian city of Chelyabinsk. As it streaked nearly sideways across the sky, it grew brighter until it was a blinding white light that cast eerie moving shadows. It radiated high-intensity light for several seconds as it elongated and grew into a larger source, then gradually slowed, dimmed, and changed color from white to yellow, orange, and then a dull red. The sources of light continued along the original path as the main body stopped, forming the roiling head, rising like thunderclouds from a long, puffy trail that traced its entire path across the heavens. As residents looked on in wonder, the trail split neatly into two parts that seemed like mirror images of one another with a sharp line between them (Fig. 1). The columns silently drifted apart and grew upward, attracting early morning office workers and students to windows to observe and snap photos with their smart phones.

Suddenly, just under a minute and a half after the brightness of the fireball peaked, an unexpected blast wave ripped through the city, blowing out windows and showering spectators with high-speed shards of glass and falling ceiling 
tiles. Nobody anticipated a blast following so long after the spectacle in the sky, the delay being due to the time it took the blast wave to travel at the speed of sound some $29 \mathrm{~km}$ down to the city. Fifteen hundred people would have to be treated for their injuries. Out on the streets, startled residents screamed and shouted expletives as the concussion hit them and set off car alarms. After the main boom, smaller explosions continued in rapid succession like artillery fire. The blasts lofted years of accumulated dust from old factories, creating a thick black pall over parts of the city as the booms continued. Power lines and mobile phone towers bounced and shook from the succession of blasts, leading to outages. To the south of the city, small black rocks rained down on villages and snow-covered fields.

Across the city and surrounding countryside, dashboardmounted digital cameras captured the event from every angle. Within minutes, computer-savvy Russians were uploading images and videos to the internet. The Russian media initially reported the event as an unidentified flying object (UFO), and these reports were forwarded across the world by users of social media. Scientists at the Jet Propulsion Laboratory (JPL) in Pasadena first learned of the event from Twitter, and only because a dedicated JPL media specialist monitors her feed after hours. Others found out from Facebook because a late-night chess-playing Canadian shared a story posted by his early morning Russian counterparts.

U.S. government satellites with instruments designed to detect nuclear explosions recorded the bright flash as it happened, but did not automatically report it because it did not have the characteristics of a weapon. Seismometers in Russia recorded the shaking ground, which resembled an earthquake. Slower-moving low-frequency sound waves in the air (infrasound), which carried the first atmospheric news of the event, had not yet reached the western border of Russia by the time the international wire services picked up the story, albeit with sketchy anecdotal descriptions.

In the western hemisphere, most relevant experts were blissfully asleep, either on the U.S. east coast, where it was after midnight, or in pre-dawn Europe. Ironically, many were prepared for what was expected to be the big news event of the day, the close passage of asteroid (367943) Duende. With interviews and press conferences scheduled, they had no way of knowing that the Chelyabinsk event had just occurred. NASA does not have a system to detect atmospheric explosions, and the International Monitoring System only puts out alerts when explosions are nuclear, so nobody's pager went off. Without fortuitous social media connections, it is possible that officials would have been caught entirely off guard when asked by reporters if the Chelyabinsk event was related to (367943) Duende, or if NASA's calculations were wrong.

Those who had already seen the YouTube videos knew immediately that this was pure coincidence. The Chelyabinsk asteroid came from the direction of the Sun in the northern hemisphere, and Duende was arriving from the southern nightside. Nevertheless, if a few scientists in western time zones hadn't had their attention drawn to it and alerted their colleagues to the east, it could have created an unfortunate embarrassment (even though it was not NASA's job to know about it, the public, media, and politicians may have perceived it differently).

Embarrassment, however, is not the worst-case scenario. If it had been cloudy in Chelyabinsk that morning, it may not have been immediately apparent to locals or outsiders that this was a cosmic airburst. The bright flash and huge blast, followed by the sound of heavy artillery, and parts of the city shrouded in dark smoke, could have been misperceived as an act of aggression. Snezhinsk, to the north, is the Russian equivalent of Lawrence Livermore National Laboratory in the U.S., and the region is of nuclear strategic importance. Russia, unlike its neighbor Kazakhstan in the direction from which the asteroid came, is still a nuclear-armed state. It is hard to know what would happen in the heat of the moment when there is great uncertainty about the cause of a halfmegaton explosion over a Russian city.

If the same asteroid had come from a different direction it may have had far worse consequences on the ground. It was lucky that it arrived on a near-grazing trajectory, because its energy was spread out over a large distance, and was deposited high enough in the atmosphere to diverge and disperse before it reached the ground. A steeply descending asteroid would explode much lower and its downward momentum would carry it closer to the ground, causing much more severe damage (Kring and Boslough, 2014). It is almost certain that there would have been massive casualties under just slightly different conditions.

Thanks to the plethora of data collected from the dashboard cameras, seismometers, infrasound detectors, and satellites, we have a very good understanding of what happened. The best estimate of the kinetic yield (explosive energy) is 400-500 kt, making Chelyabinsk the most powerful such event observed since the 1908 Tunguska explosion [3-5 Mt (Boslough and Crawford, 2008)]. Analysis of video combined with subsequent onsite stellar calibrations enable reliable estimates of entry velocity ( $\left.19 \mathrm{~km} \mathrm{~s}^{-1}\right)$, angle ( $17^{\circ}$ elevation), and altitude of peak brightness $(29 \mathrm{~km})$. This implies a preentry diameter of $\sim 20 \mathrm{~m}$ and mass of $\sim 12,000 \mathrm{t}$. Satellite sensors recorded the emission peak at 03:20:33 UT, with a total radiated energy of $3.75 \times 10^{14} \mathrm{~J}(\sim 90 \mathrm{kt})$. A typical bolide luminous efficiency of $20 \%$ implies a total energy of $\sim 450 \mathrm{kt}$, consistent with infrasound and other observations. The maximum radiant intensity was $2.7 \times 10^{13} \mathrm{~W} \mathrm{sr}^{-1}$, corresponding to an absolute magnitude (referenced to a distance of $100 \mathrm{~km}$ ) of -28 , or more than $30 \times$ brighter than the Sun as seen by an observer located directly below (Brown et al., 2013).

The shallow entry angle led to a long bolide duration (16.5 s) and energy was deposited over hundreds of kilometers, leading to an extended, near-horizontal, linear explosion. The blast was distributed over a large area, and was much weaker than it would have been in the case of a steep entry and a more concentrated explosion closer to the surface. The orientation also led to different phenomena than expected for a more vertical entry. There was no ballistic plume as observed in the case of the Shoemaker-Levy 9 impacts into Jupiter $\left(45^{\circ}\right)$, or calculated for Tunguska $\left[\sim 35^{\circ}\right.$ (Boslough 
and Crawford, 1997)]. Instead, buoyant instabilities grew into mushroom clouds and bifurcated the trail into two contrarotating vortices (Kring and Boslough, 2014).

Hydrodynamic models can now be initialized with extremely accurate energy depositions at correct locations, and results can be compared with observations (such as timing and distribution of blast energy at the surface, and evolution of the trail) to validate the models and better understand the physical phenomena associated with airbursts. Models suggest that airbursts are more damaging than nuclear explosions of the same yield (traditionally used to estimate impact risk, although nuclear radiation is not produced by the impact of a NEO). On the basis of recent observation-based size-frequency data, Chelyabinsk was estimated to be a once-per-century event while Tunguska was about once-per-millennium. These two events suggested that the size-frequency data underestimated the frequency of large airbursts. However, one of us (Harris of MoreData! Inc.) has updated the size-frequency estimates and determined that Chelyabinsk is a 50-year event. Chelyabinsk can no longer be considered an outlier as it is the only observed event of its size in the last 50 years. On the other hand, Tunguska is now estimated to be a 500-year event, so its occurrence just over 100 years ago can still be considered a statistical outlier. The new size-frequency data are presented and discussed in section 3 (see also Table 1). The risk from airbursts is greater than previously thought, but still highly uncertain.

There are several approaches that can be employed to reduce the uncertainty in the risk, and also to reduce the risk itself. One way to reduce uncertainty is to improve the monitoring and quantification of bolides. There are extensive records of past bolides seen by U.S. government satellites, and these data have been used by Brown et al. $(2002,2013)$ to estimate the flux of $1-\mathrm{m}$ - to 10 -m-diameter asteroids (permission has now been granted for the scientific community to access more recent data; see http://neo.jpl.nasa. gov/fireball). Another approach would be to launch dedicated satellites with missions optimized to observe and quantify bolides. Such a system would also provide immediate notice and location of an airburst event, which would aid in communication to the public as well as recovery of meteorites.

The best way to reduce the risk from Chelyabinsk-class asteroids would be to implement a short-warning survey, with groundbased telescopes optimized to search the entire night sky on a roughly daily basis, which would find even asteroids and meteoroids much smaller than Chelyabinsk as they brighten during the last days or weeks of their final encounters with Earth (see section 5). An alternative or complementary measure could be an infrared space telescope with a position and cadence optimized to find small objects within days or weeks of impacts. Such systems would also find smaller objects that are not a threat, but that would be a potential boon to science, and could even be commercialized with costs recouped by the recovery of meteorites and high-end adventure tourism (Boslough, 2014). If such a system had existed in 2013, the storyline of Chelyabinsk might read differently:
On February 15, 2013, at the crack of dawn, a glowing object appeared as expected in the clear southeastern sky seen from the industrial Russian city of Chelyabinsk. As it streaked nearly sideways across the sky it was tracked with scientific instruments, including telescopes, spectrometers, high-definition cameras, radar, and calibrated radiometers. Optical pyrometers determined the temperature of the fireball as it cooled and changed color. As the spark-like fragments continued to fade out and fall in dark flight, they were still glowing in the thermal infrared and were tracked all the way to the ground, where they were immediately recovered and taken to meteorite laboratories that had been prepared for them.

Residents who had been warned to stay away from windows and to watch from a safe distance looked on in wonder. Hotels in outlying areas were filled with adventure tourists who had come from all over the world to see this once-in-a-lifetime spectacle. Some had volunteered to join scientific expeditions to scour the fall area for meteorites.

Researchers were able to use the wealth of highquality, calibrated data to improve models of ablation, fragmentation, breakup, and explosion, improving their understanding of the high deformation-rate processes that will ultimately improve risk assessment, as well as facilitate the design of more effective impulsive deflection methods if a larger hazardous asteroid is discovered with sufficient advance warning.

\section{CURRENT UNDERSTANDING OF THE IMPACT HAZARD}

Although the impact frequencies for NEAs with diameters, D, larger than $100 \mathrm{~m}$ are known quite well, and about $90 \%$ of NEAs with D $>1 \mathrm{~km}$ have been discovered, smaller NEAs are less well characterized. Relative to astronomical surveys of NEAs, actual impact events like Chelyabinsk (although small in number), and recalibration of infrasonic airwave data (Silber et al., 2009), suggest that the frequencies of impacts by bodies tens of meters in diameter may be several times higher than those estimated earlier from optical survey data.

Our present understanding of the numbers of NEAs and impact probabilities is summarized in Fig. 2, in which the curve traced by the circles represents current best estimates of the cumulative number of NEAs larger than a given diameter. For a given $\mathrm{H}$-value interval, optical survey population estimates are derived by counting the number of redetections of previously discovered objects compared to the total number of detections (new discoveries plus redetections) in the most recent survey period of two years. As the ratio of redetections to all detections approaches $100 \%$, so does the survey completion. However, since some asteroids are easier to find than others, and the easy ones are discovered first, the estimated completion is actually always less than the redetection ratio. A correction to the completion estimate is derived using a realistic model of the NEA population and 


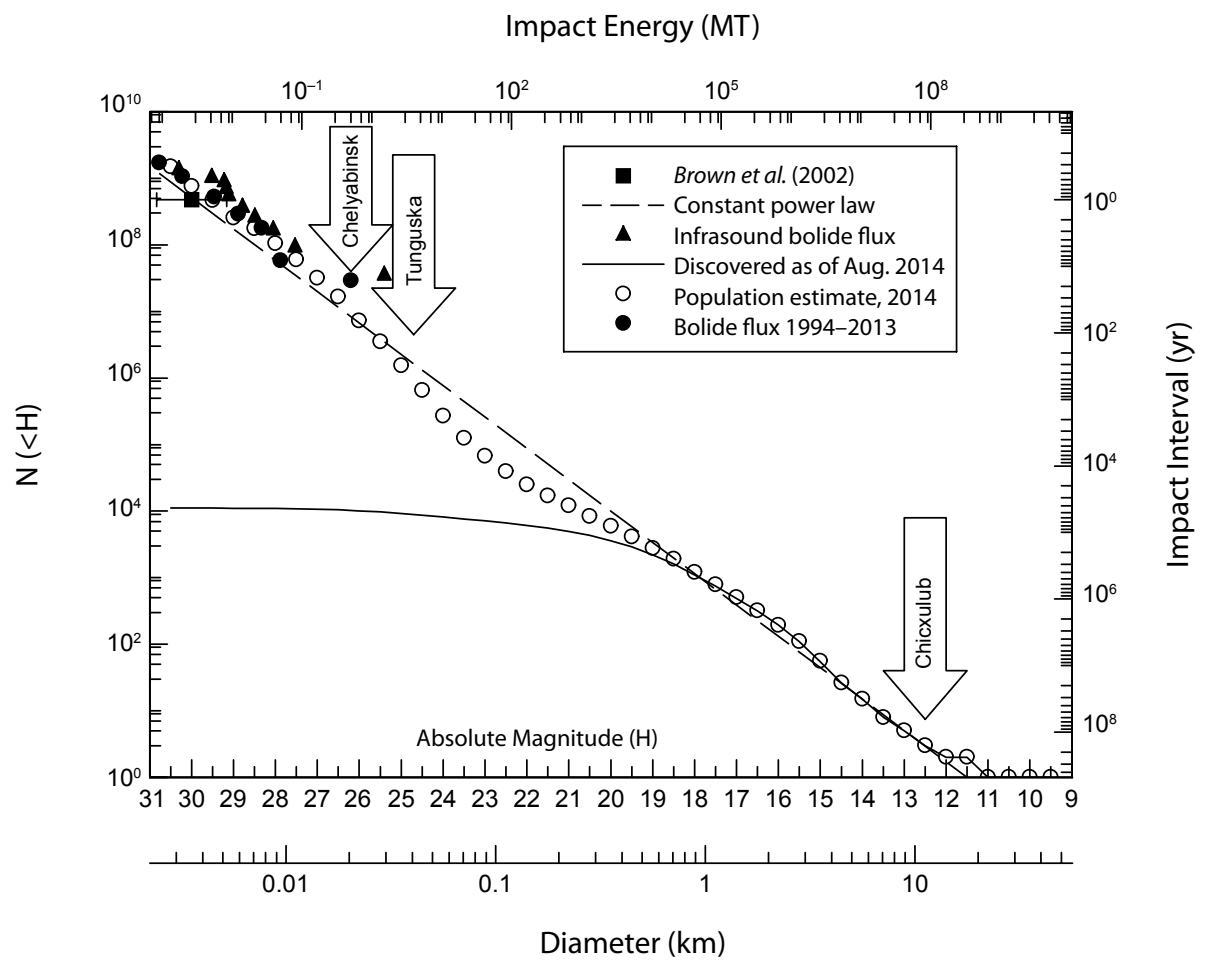

Fig. 2. Estimated number of NEAs larger than a given diameter/brighter than a given absolute magnitude. The continuous curve represents discoveries as of August 2014; the curve traced by the circles represents the estimated total number of objects in the NEA population, derived from computer modeling of discovery and redetection rates by optical telescope surveys over the two-year period to August 2014. The population estimated in this way appears to be in excellent agreement with population estimates from bolide and infrasound data. The estimated mean interval between impacts of objects larger than a given diameter can be read off on the righthand scale using the curve traced by the circles. The conversion from $\mathrm{H}$ to diameter assumes an albedo of $\mathrm{p}_{\mathrm{V}}=0.14$. The uncertainty in the estimated population curve in the range $\mathrm{D}=\sim 10$ to $\sim 100 \mathrm{~m}$ from all errors, observational, model, and the albedo assumption is probably less than a factor of 3 . At larger sizes, the uncertainty is even less, diminishing to only a few percent for $D \sim 1 \mathrm{~km}$ and larger.

a computer simulation of a hypothetical optical telescope survey over the same time period, in this case 20 years.

The latest update of the NEA population, presented in Fig. 2, appears to largely resolve the above-mentioned discrepancy in population estimates between astronomical surveys and bolide frequencies (Brown et al., 2013; Silber et al., 2009). Earlier population estimates from astronomical surveys obtained a population in the tens of meters size range about a factor of 3-4 lower than estimated from bolide and infrasound data (see, e.g., Harris, 2014). However, the most recent population estimate falls within the scatter over the size range of the bolide estimates.

To the best of our knowledge there is no current threat from any NEA larger than about $4 \mathrm{~km}$ in diameter, since they have probably all been found and none are on a threatening trajectory during the next century. There remains a very small but uncertain threat from large comets. Objects originating in the Oort cloud are discovered typically only about two years prior to perihelion, precluding any effective spaceborne de- flection action in these cases. For example, Comet C/2013 A1 (Siding Spring) was discovered in January 2013 on its way into the inner solar system from the Oort cloud. Early orbit calculations revealed a small probability that the comet would impact Mars on October 19, 2014, with a velocity of some $56 \mathrm{~km} \mathrm{~s}^{-1}$. Even with a diameter of around $500 \mathrm{~m}$, at the lower end of initial size estimates, an impact of the comet on Mars would have had dramatic, possibly global, effects, not to mention the potentially disastrous consequences for the valuable scientific assets on its surface and in orbit around it. Later refinements of the orbit of C/2013 A1 ruled out an impact but showed it would pass within around 20 martian diameters of the planet (Farnocchia et al., 2014).

Apart from the frequency of impacts by cosmic bodies of various sizes, a major question concerns the scale of damage such an impact might cause. The Chicxulub (K-Pg boundary) impact at $66 \mathrm{Ma}$ presents a qualitative example of how very infrequent impacts by large NEOs can greatly change the global ecosphere and biosphere of Earth. Near- 
Earth objects with $\mathrm{D}=2-3 \mathrm{~km}$, and possibly as small as $1 \mathrm{~km}$, could destroy civilization as we know it, although the degree to which that civilization is robust or vulnerable to the multiple environmental effects of such impacts remains speculative. Impacts of bodies only $500 \mathrm{~m}$ in diameter could, for example, severely damage or destroy the ozone layer (Birks et al., 2007). Near-Earth objects of such sizes impacting in the ocean could also produce very destructive tsunami, although a number of studies (e.g., Gisler et al., 2011; Wünnemann et al., 2007; Korycansky and Lynett, 2005; and references therein) suggest that such waves would largely disperse before reaching coastlines, unless the impact were rather close to the coastline. The environmental consequences of damage from a ground explosion are simulated in the online program Impact Earth [(http://www.purdue.edu/ impactearth/ - for details, including assumptions and uncertainties, see Collins et al. (2005)]; how much infrastructure would be destroyed or how many lives would be lost would depend on proximity to the impact (see, e.g., Garbolino and Michel, 2011) and how much advance warning there was to strengthen infrastructure or evacuate the region.

Recently there has been emphasis on the effects of the smaller but much more frequent impacts of NEOs several tens of meters in diameter and smaller. A big issue has been, what is the smallest NEO that would be damaging? The NASA NEO Science Definition Team study (NASA SDT) (Stokes et al., 2003) concluded that NEOs with D $<40 \mathrm{~m}$ would explode rather harmlessly in the upper atmosphere, but that conclusion has been undercut for several reasons. First, Boslough and Crawford (2008) realized that downward momentum of a NEO through the atmosphere would be more damaging than a stationary explosion, and concluded that the great damage at Tunguska could have been produced by a body only $40 \mathrm{~m}$ in diameter (a few megatons of equivalent energy). Second, we have learned that some types of smaller projectiles can be more damaging than the average, such as nickel-iron objects, which are relatively dense, and objects that can reach the ground by virtue of unusual aerodynamic shapes. Low cosmic velocities (12-14 $\mathrm{km} \mathrm{s}^{-1}$ ) and shallow entry angles also contribute to reducing aerodynamic stress and may allow meter-sized stony meteoroids to reach the ground, such as the object that caused the 13-m-diameter impact crater near the Peruvian village of Carancas in 2007 (Kenkmann, et al., 2009; Tancredi et al., 2009). The Chelyabinsk event (section 2) could have been even more destructive at ground zero if it had entered the atmosphere at a less shallow angle. On the other hand, the impact was unfortunately close to a major city, which would not usually be the case. One factor that needs to be recognized is that any nominal estimate of size (and associated damage potential) has uncertainties that could be very great for a body discovered only shortly before impact. Asteroids have diverse compositions and shapes, wide ranges of albedo and bulk density, and other uncertain attributes, which could be difficult or impossible to assess before impact but conceivably could result in the nominal estimate of destructive energy being in error by an order of magnitude in either direction.
Thus, even if a body just $10 \mathrm{~m}$ in diameter or smaller were predicted to strike a locality, a prudent civil defense official might well warn people to evacuate, or at least stay indoors in structurally sound buildings well clear of windows (to avoid flying glass), to cover their ears (to avoid eardrum rupture), and, where possible, to open all windows and doors (to minimize damage by the overpressure of the blast waves).

The general approach to dealing with the NEO hazard is to (1) search the skies for NEOs that may strike Earth; (2) plan and carry out a deflection spacecraft mission if there is sufficient time and the threat is sufficiently great (high probability of damaging impact); and/or (3) evacuate or otherwise prepare to mitigate the effects of an impact.

Telescopic searches for NEOs are of several kinds (see the chapter by Jedicke et al. in this volume). The Spaceguard Survey was designed to find $>90 \%$ of NEOs $>1 \mathrm{~km}$ in size, and it has accomplished that task, although taking some years longer than the mandated decade. As a byproduct, the Survey has found many NEOs down to hundreds of meters in diameter, and a statistical sample of those down to $\sim 10 \mathrm{~m}$ in diameter. The Survey continues, although with modifications. The NASA NEO Science Definition Team report and the subsequently passed law by the U.S. Congress (George E. Brown, Jr. NEO Survey) recommend finding $90 \%$ of NEOs with diameters larger than $140 \mathrm{~m}$ within the near term. Various groundbased, e.g., the Large Synoptic Survey Telescope (LSST), and spacebased, e.g., the NEOCam and Sentinel, projects are designed to meet this goal, although the timeframe for the surveys keeps slipping into the future. Yet another approach is to use dispersed small telescopes to cover the sky on a nightly basis and find NEOs as they approach Earth for the last time before striking. The Catalina Sky Survey, for example, found the tiny asteroid/meteoroid $2008 \mathrm{TC}_{3}$ just over 20 hours before it struck in the Sudanese desert. Several systems are under development [e.g., the University of Hawaii's Asteroid Terrestrial-impact Last Alert System (ATLAS) or ESA's Fly-Eye concepts — see section 5] that could find up to half of potential impactors during the days to months before impact - those coming from the night sky rather than from the daylit sky — providing adequate time for evacuation warnings.

If a sizeable NEA is discovered to be on a possible impact trajectory years or a decade or two before impact, then a spacecraft mission designed to deflect the asteroid is technically feasible. Direct deflection of a hazardous NEA so that it passes several Earth radii away from Earth instead of striking Earth may be necessary in the worst case. However, an asteroid may closely approach Earth on a previous apparition so that the perturbation by Earth's gravitational field is just the right amount to cause its orbit to enter a resonance condition with Earth's orbit and impact Earth on a later approach. The small region of space through which the NEA has to pass to enter a resonance is called a gravitational (or resonance) "keyhole" (see the chapter by Farnocchia et al. in this volume). A recent prominent example was the prediction of a keyhole only about $600 \mathrm{~m}$ wide in the case of the 2029 approach to within 35,000 km of Earth's surface of 
(99942) Apophis, a NEA with a diameter of some $325 \mathrm{~m}$. If Apophis were to pass through the keyhole an impact with Earth would follow in the year 2036 (see, e.g., Yeomans et al., 2009). Fortunately, recent observations have ruled out a possible passage of Apophis through the keyhole. Accurate knowledge of an asteroid's orbit allows the existence of keyholes for the object to be predicted well in advance. It is much easier to deflect the asteroid from passing through a small keyhole than having to deflect it by several Earth radii.

Studies of deflection missions are in their infancy (see section 4) but there appear to be no fundamental issues preventing existing technology from being put together rapidly to build a deflection mission, should the need arise. Similarly, the disaster response community has little familiarity with asteroid impact as a natural disaster, but the kinds of environmental effects arising from the atmospheric explosion or ground impact of a NEO are similar to those of more common natural disasters (Garshnek et al., 2000) so that, provided a predicted impact is properly communicated to relevant officials in the relevant nations or localities, it seems likely that appropriate responses to a predicted impact would result in most cases. There will always be, though diminishing with time as surveys advance, the chance that an unpredicted impact will occur, as in the case of Chelyabinsk.

\section{NEAR-EARTH ASTEROID DEFLECTION TECHNIQUES}

Many ideas have been proposed to deflect an asteroid from an Earth-bound trajectory or to disrupt it. However, to date none has been tested in space and the feasibility of proposed techniques given present technology differs greatly.

Three apparently realistic methods have emerged from the numerous studies devoted to this topic, namely the kinetic impactor, the gravity tractor, and blast deflection, which are described below. Other suggested techniques include directing a laser beam at the asteroid's surface to expel (ablate) material from it, thereby creating a force that moves the object so as to conserve linear momentum (Gibbings et al., 2013). Similarly, the use of large mirrors or lenses to concentrate the Sun's energy onto an asteroid using single or multiple spacecraft has been advocated. Another idea would be to exploit the momentum transmitted by a collimated beam of ions impacting the asteroid surface (Bombardelli et al., 2013). Ion thrusters attached to a hovering spacecraft could be used for this purpose. The spacecraft, placed at a distance of a few asteroid diameters, would also need an ion thruster pointed in the opposite direction of the asteroid to balance the spacecraft's reaction to the deflecting thrust and keep a constant distance between the asteroid and the shepherd spacecraft. A more speculative idea is to paint the surface of an asteroid to increase its albedo, thereby enhancing the solar radiation pressure acting upon it (Paek, 2012). These methods require much a priori knowledge of the asteroid and/or they involve technologies that require significant further development. In particular, providing adequate power for deploying beams of light or ions to deflect a threatening NEA to a safe trajectory, developing systems that can operate continuously and reliably over periods of years, and the requirement for autonomous guidance and control of the deflecting spacecraft are all major technical challenges.

Most recent studies (e.g., Shapiro et al., 2010) find that the most reasonable approach in the vast majority of cases, especially for NEAs with diameters above about $100 \mathrm{~m}$ (see section 6), is deflection by hypervelocity kinetic impact by a massive spacecraft, while an observer spacecraft (preferably with a slow push or pull capability, such as a gravity tractor, as discussed below) looks on to assess the success and magnitude of the deflection. The impact of the artificial projectile (spacecraft) transfers momentum to the NEA, causing a small change in velocity. If a large amount of ejecta is produced by the impact event then the momentum transferred can be greatly enhanced, as dictated by the law of conservation of momentum, resulting in a larger deflection of the object's course. The production of ejecta increases the effectiveness of impulsive deflection techniques but the amount of ejecta produced depends strongly on the subsurface properties of the asteroid. A series of kinetic impactors should suffice for threatening NEAs possibly up to $1 \mathrm{~km}$ in size.

The NASA Deep Impact mission (A'Hearn et al., 2005) successfully caused an impactor to collide with the nucleus of Comet 9P/Tempel 1 on July 4, 2005. Due to the large mass of the $6-\mathrm{km}$-diameter nucleus, and nongravitational forces acting on it, it was not possible to measure a change in the comet's trajectory resulting from the impact, so the experiment did not provide a measurement of momentum transfer to the comet. Nevertheless, the mission successfully impacted its target and highlighted the complex autonomous guidance necessary to do so.

The kinetic impactor technique has been the subject of a number of studies carried out by major space agencies. In 2006 the European Space Agency funded and coordinated detailed studies of a kinetic impactor concept called Don Quijote, which consisted of an orbiter and a small impactor spacecraft arriving several months later than the orbiter (e.g., see Wolters et al., 2011, and references therein). The orbiter would first characterize the target and measure the deflection due to the impact of the second spacecraft. While the Don Quijote concept as such has not been funded to date, it has served as the inspiration for aspects of the NEOShield program and European participation in the AIDA concept. In early 2010, ESA, Johns Hopkins University’s Applied Physics Laboratory, NASA, the Côte d'Azur Observatory, and the German Aerospace Center initiated a study of AIDA, the aim of which is to deflect the small secondary of a binary asteroid, chosen so that the perturbation to the orbit of the secondary can be observed from groundbased facilities in 2022. The target is the binary asteroid (65803) Didymos. The mission (Cheng et al., 2015; Michel et al., 2015) has two independent components: the projectile spacecraft, "Double Asteroid Redirection Test" (DART), which would be developed in the U.S., and the European rendezvous spacecraft Asteroid Impact Mission (AIM). DART would serve as a test of our ability to impact a small (150-m-diameter) object, while 
AIM would allow it to be characterized in detail by observing the target before, during, and after the impact event. The advantage of the AIDA concept, compared to a mission to deflect a normal NEA, lies in the relative ease with which the orbit of a small binary moon around the primary can be changed to a measurable extent, and the fact that in the case of Didymos, due to the favorable observation geometry, the change can be measured by groundbased telescopes monitoring the variability of reflected sunlight caused by eclipses and occultations in the binary system. While groundbased observations would complement the observations made by AIM and provide a vital backup, AIM would measure the resulting change in orbit of the moon with greater accuracy, and would provide detailed in situ reconnaissance of the system's physical characteristics. The results of the AIDA mission would allow the detailed verification of impact models on a realistic size scale. At the time of this writing AIDA is the only deflection mission concept that is actively under study by major space agencies. If funded for launch, the impact would occur in October 2022.

An alternative, relatively inexpensive, test of the kinetic impactor concept, under consideration in ongoing NEOShield work, would be to impact a NEA far from its rotation axis, thus causing a change in its rotation rate, which, depending on the choice of the target, could be measurable with groundbased telescopes (NEOTWIST, Drube et al., in preparation). The change in rotation rate would provide insight into the same near-surface structural characteristics on which the efficiency of the kinetic impactor deflection concept depends. A possible target for such a test would be the NEA (25143) Itokawa, which has been well studied by the Japanese Hayabusa mission. The payload of the impactor spacecraft could include an ejectable science package to allow the impact to be observed and provide information on the crater formation, and ejecta mass and velocity distributions. While any type of deflection test would be very instructive, given the diverse properties of NEAs we should bear in mind that the responses to impacts could be very different for other NEAs.

In the case of the gravity tractor ( $L u$ and Love, 2005), a spacecraft under power hovers near an asteroid and uses the gravitational attraction between it and the asteroid to tow the asteroid off course. Multiple spacecraft flying in formation could be deployed to expedite the gravity tractor maneuver and/or provide redundancy (Foster et al., 2013). Given the very small tractor/asteroid mass ratio, this technique is only useful for small objects, but since small NEAs are more numerous than larger ones, a gravity tractor might suffice for the most likely scenarios in which deflection seems to be warranted, depending on the available time before the predicted impact. How small is still a matter of debate, but the current practical upper diameter limit suggested by recent (as yet unpublished) NEOShield results is around $50 \mathrm{~m}$ for a direct impact trajectory [Shapiro et al. (2010) give roughly $100 \mathrm{~m}$ for the same limit]. The main difficulty is developing reliable autonomous control systems able to keep the tractor at a fixed distance from a small, rotating, irregularly shaped asteroid, possibly for a period of many years. If time is insufficient for the use of a gravity tractor as sole deflector, a currently realistic scenario is deployment of the gravity tractor as a secondary deflection technique to an impulsive primary strike. For example, an appropriately designed and instrumented spacecraft could first act in a reconnaissance capacity and study the NEA in advance of the impact, subsequently remain in place to monitor the deflection, and have the capability to finally act as a gravity tractor to adjust the post-impact trajectory of the NEA, if necessary. Alternatively, if the threatening object were due to pass through a keyhole, then a relatively small deflection, which could be performed by a gravity tractor alone, would suffice to cause the object to miss the keyhole. In fact, in the case of keyhole avoidance, the gravity tractor alone may suffice to deflect much larger asteroids.

While the gravity tractor appears to be feasible with current technology, the slow-push alternatives, such as the ion-beam shepherd and laser ablation mentioned above, may in time prove to be more practical and effective per kilogram of launch mass in many cases, depending on future developments of the relevant technologies and the details of the hazard scenario. An important advantage of the gravity tractor is that few characteristics of the NEA need to be known in advance. However, gravity tractors and the alternative slow-push techniques are weak, so depending on the time available and the circumstances of the NEA's orbit (presence of keyholes), the much more powerful capability of a kinetic impactor may be desirable or necessary.

In the very unlikely case of a very large hazardous asteroid and/or inadequate time for the deployment of other deflection techniques, "blast deflection" with a nuclear device may offer the only technically feasible solution: This technique requires the use of an explosive close to, on the surface of, or buried beneath the surface of the NEA. Complete disruption of the threatening object would be an option if it were small enough so that the largest pieces of debris would not present a hazard on impact. Otherwise, the blast should be designed to deflect the asteroid, not break it up. The blast from a nuclear device would cause the outer layers of the NEA to evaporate and be expelled from the surface. The effect would be similar to rocket propulsion, thus altering the asteroid's trajectory. Burying an explosive on the asteroid before setting it off, or delivering the device by means of a surface penetrator, would probably be most effective at ejecting surface material, but this method would require prior knowledge of the material properties and subsurface structure of the object. A deflection mission concept study supported by NASA's Innovative Advanced Concepts Program combines a kinetic impactor mission with a nuclear device for deflection of large asteroids, or for cases in which there is very little warning time. In the very unlikely case of a large comet, deflection would be very challenging at best due to the likely short lead time and nongravitational forces affecting the comet's trajectory. In any case, political and legal objections, not to mention technical issues, would have to be overcome before nuclear explosive devices could be used for NEA deflection. 


\section{CURRENT PRIORITIES AND INITIATIVES}

At the time of this writing there are a number of groundbased telescopes under development with the goal of discovering very small NEOs to provide advance warning of about one week for objects with diameters in the range 30-50 m, and longer times for larger objects. Examples of such wide-field, rapid-scan search programs currently under development are ESA's Fly-Eye (Farnocchia et al., 2012) and NASA's ATLAS (Tonry, 2011). Plans for ATLAS include up to eight small telescopes, each fitted with cameras of up to 100 megapixels. According to the ATLAS team, the system should offer a warning of about 1 week in the case of a 50-m-diameter asteroid or "city killer," and 3 weeks for a 150-m "small country killer." Such wide-field survey systems will complement the established surveys, such as the Catalina Sky Survey and Pan-STARRS, in being able to provide warning of small objects in the final phase of their plunge to Earth, thereby enabling measures on the ground, such as evacuation, to be carried out.

Apart from the work to discover and track NEOs, there is much ongoing groundbased effort to investigate their physical properties. Observations in the visible and infrared spectral regions of light reflected from asteroid surfaces are a powerful means of studying their compositions and assigning them to taxonomic classes. For reviews of earlier work the reader is referred to Binzel et al. (2002), and for more recent developments, to the chapters in this volume, such as those by Binzel et al. and DeMeo et al. Efforts to derive information on the physical properties of asteroids by observing absorption features in reflected sunlight are complemented by observations of thermally emitted infrared radiation and radar investigations. The latter two approaches, briefly discussed here, have brought major advances relevant to the impact hazard since the publication of Asteroids III.

A survey of the sizes and albedos of more than 100,000 asteroids has been carried out by the NASA WISE space telescope (Wright et al., 2010). WISE was launched to Earth orbit in December 2009 carrying a 40-cm-diameter telescope and infrared detectors. WISE surveyed the sky for 12 months and the objects observed included a total of at least 584 NEOs, of which more than 130 were new discoveries (Mainzer et al., 2011). The specially funded Near-Earth Object Wide-field Infrared Survey Explorer (NEOWISE) program analyzed images collected by the WISE spacecraft to derive information on the NEOs detected. The fact that the cryogenic phase of the WISE mission measured asteroid thermal emission in up to four infrared bands, centered on $3.4,4.6,12$, and $22 \mu \mathrm{m}$, allowed reliable values of diameter, albedo, and other parameters to be derived for many of the asteroids observed. Mainzer et al. (2011) report that after debiasing with a synthetic NEO population, the NEOWISE results imply a total of some 980 for the number of NEOs larger than $1 \mathrm{~km}$. These results mean that the Spaceguard goal of detecting $90 \%$ of all NEOs with diameters larger than $1 \mathrm{~km}$ has now been met (see the chapter by Mainzer et al. in this volume). A complementary program to NEOWISE,
"ExploreNEOs" (Trilling et al., 2010; see also the chapter by Mainzer et al. in this volume), has used the Spitzer Space Telescope to observe some 600 previously detected NEOs to study the size and albedo distributions of the NEO population, and identify NEOs that may have a cometary origin or that could serve as accessible and scientifically interesting targets for future robotic or manned space missions.

Following in the footsteps of the successful Infrared Astronomical Satellite (IRAS) mission in 1983, the WISE and Spitzer missions have highlighted the value of spacebased infrared telescopes for basic physical characterization of the asteroid population. In addition to size and albedo information, application of thermal models to thermal-infrared observations has revealed their potential to provide information on thermal inertia and mineralogy, including the presence of metal on the surfaces of asteroids (Harris and Drube, 2014). A threatening NEA containing a large amount of metal would presumably be relatively robust and massive, depending on its internal structure, factors that would require careful consideration by deflection-mission planners and/or those mandated to manage mitigation, e.g., evacuation and other activities on the ground in advance of a possible impact. Moreover, the identification of NEAs with high metal content is an important task for endeavors in the field of planetary resources. Metal-rich asteroids could provide a vast reservoir of vital materials for future interplanetary space activities and perhaps eventually for use on Earth.

The success of NEOWISE suggests that future missions with more sensitive spacebased infrared telescopes, designed specifically for NEO detection, would bring major advances in our knowledge of the mitigation-relevant small end of the NEO size distribution, and may even provide a useful guide for interplanetary prospectors. Examples of such spacebased infrared telescopes currently under study are the Near-Earth Object Camera (NEOCam) and Sentinel (see the chapter by Jedicke et al. in this volume). In particular, a spacebased infrared telescope on a heliocentric orbit inside that of Earth, such as the Sentinel spacecraft, would have an advantage over Earth-based telescopes in detecting Aten asteroids and inner-Earth objects (Atiras), which spend much of their time in the daylit sky as seen from Earth.

Radar is a crucial means of obtaining very accurate astrometric data for NEOs. Radar observations can provide enormous improvements in the precision of a NEO's orbit and allow the position of the object to be projected much further into the future than allowed by optical monitoring alone. The uncertainty in the prediction of a future impact can be dramatically reduced with the help of radar data or, much more likely, a possible impact can be ruled out (Ostro and Giorgini, 2004). Radar is also a powerful method for the characterization of NEOs, especially their sizes, shapes, and surface structure. A radar echo contains information not only on the position and velocity of a NEO, but also on a number of mitigation-relevant physical parameters. Radiation transmitted at a single frequency is returned from a rotating asteroid with a spread of (Doppler-shifted) frequencies, each component frequency being associated with a particular time 
delay depending on the distance to the reflecting surface element. The "delay-Doppler" distribution of echo power is determined by the size, spin rate, orientation, and shape of the target asteroid, and radar reflectivity of the surface material. Given a suitable transmission coding, the information in a radar echo can be processed to provide resolved radar "images" of asteroids. The strength of the echo, normalized to the size and distance of the target ("radar albedo"), can provide information on the mineralogy of the asteroid surface, in particular its metal content. A circularly polarized transmission will give rise to a mix of "opposite sense" (OC) and "same sense" (SC) polarized radiation in the echo, depending on the number of reflections taking place at the surface. The ratio of SC/OC polarization in the echo is an indicator of the roughness of the surface at the scale of the radar wavelength (typically 3-13 cm). In particular, radar observations have observed a large number of binary NEAs, and have taught us that small asteroids can have very irregular shapes but also that some NEAs are surprisingly round, with equatorial bulges, suggestive of loose agglomerates of boulders and gravel that change shape as the spin rate exceeds a critical value, presumably preceding the spinning off of material that may then accumulate to form a moon (see the chapter by Walsh and Jacobson in this volume). For an overview of radar observations of asteroids, see the chapter by Benner et al. in this volume.

Rendezvous missions are a valuable source of mitigationrelevant physical information on particular NEAs. A concept under study by NASA at the time of writing is the Asteroid Redirect Mission (ARM), in which either an asteroid with a diameter of less than $10 \mathrm{~m}$ is captured whole, or a 2-3-m boulder from a larger NEA is collected, and brought into lunar orbit (see the chapter by Abell et al. in this volume). For the second option, NASA is considering a test of the gravity-tractor concept by using the combined mass of the spacecraft and the boulder to slightly change the orbit of the mother NEA. In general, regardless of which option is chosen, experience gained with ARM in the rendezvous and approach phases, and in capturing and maneuvering large masses with a solar-electric powered spacecraft, will be of direct benefit to mission planning for planetary defense.

While their primary aim is to contribute to our understanding of solar system history, sample-return missions to asteroids also provide valuable information for planetary defense. The Japanese Hayabusa mission was the first mission to return samples of an asteroid to Earth. The micrometer-sized grains returned by Hayabusa from the NEA (25143) Itokawa allowed a direct link to be demonstrated between asteroids of $\mathrm{S}$ taxonomic type and the LL class of meteorites (Nakamura et al., 2011). JAXA launched the Hayabusa-2 mission (see, e.g., Tsuda et al., 2013) on December 3, 2014, with the aim of returning a sample from the primitive (i.e., relatively unprocessed) C-class NEA (162173) Ryugu (1999 JU3), which has a diameter of about $750 \mathrm{~m}$. The payload includes a small copper projectile designed to impact the surface of the NEA at about $2 \mathrm{~km} \mathrm{~s}^{-1}$, and a small camera to observe the event. Observations in real time of the production of a crater would provide data of direct relevance to deflection studies. In addition, a small European lander, the Mobile Asteroid Surface Scout (MASCOT), will perform in situ compositional measurements. NASA is also developing a sample-return mission called the Origins Spectral Interpretation Resource Identification and Security-Regolith Explorer (OSIRIS-REx) (Lauretta et al., 2012). Launch is currently scheduled for September 2016. The sample mechanism is designed to collect between $60 \mathrm{~g}$ and a few kilograms, depending on the surface properties of the target, namely the primitive B-type NEA (101955) Bennu, which has a diameter of $\sim 450 \mathrm{~m}$.

It is important not only to improve our understanding of the mitigation-relevant physical properties of small NEAs, but also to develop technically and financially realistic test missions to enable deflection concepts to be tried out on real NEA targets, representative of the sort of objects that could threaten Earth in the not-too-distant future. Given the diverse observed shapes and mineralogies, and strong evidence for relatively low bulk densities, high porosities, and loose rubble-pile structures among NEAs, demonstrating that we can actually measurably change the orbit of a NEA is a vital step in building confidence that we can defend our civilization from a natural hazard that will otherwise cause serious loss of life and property in the future, if not threaten our longterm survival on planet Earth. The NEOShield project has performed industrial studies of test missions for the kinetic impactor, gravity tractor, and blast deflection concepts, as well as studies of the future evolution of NEA orbits after deflection attempts. While an actual deflection demonstration mission was financially beyond the scope of the NEOShield project, the aim was to provide the first designs of appropriate demonstration missions, sufficiently detailed to facilitate the rapid development of such a mission in subsequent rounds of project funding in a European/international frame.

Results from the type of studies carried out by NEOShield and similar programs obviously also serve to reduce the scientific and technical preparatory work required to bring an appropriate and viable deflection mission to the launch pad in an emergency situation.

\section{WHAT WE DO NOT KNOW: PRESSING AREAS OF CONCERN}

Although it will happen one day, the likelihood of any generation being confronted with a threatening object with a diameter, D, of around $300 \mathrm{~m}$ or larger is extremely small. In any case, NEO survey programs may provide several decades of warning time in which to prepare a response, although at present an estimated $40 \%$ of such objects remain to be discovered. Therefore, in the case of large objects, we should normally have sufficient time between hazard identification and the predicted time of impact to physically characterize the NEO using Earth-based telescopes and rendezvous missions, and prepare and test an appropriate deflection mission. The lower size threshold below which we could safely ignore an approaching object is subject to debate (see below). Even a NEO with a diameter of just $20 \mathrm{~m}$ can 
cause serious damage, impacting on average about once or twice per century. It would therefore seem prudent to take precautionary measures well in advance, and not wait until a threatening object is identified. Apart from civil defense planning in case a late detection precludes a deflection attempt, appropriate measures include investigations of the mitigation-relevant physical properties of small NEOs, such as composition, density, porosity, internal structure, spin rate, and shape, and the development and testing of appropriate and reliable deflection techniques. Unfortunately, our current knowledge of the NEO population is very biased toward the large end of the size range, since large NEOs are brighter and more readily detected and observed. Relatively little is known about the small members of the population and much work remains to be done in exploring their deflection-relevant physical properties.

At present we have no clear idea of how the international community would respond to the announcement of a credible threat from a NEO identified as significantly hazardous. After discovery of the NEO in question and initial orbit refinement, the threat could be expressed only as a probability for those parts of Earth lying on the "risk corridor" (the track of possible impact points allowed by imperfect knowledge of the object's trajectory; see Fig. 3). We would not know for sure whether the object would impact or not due to the observational uncertainties in the orbit and factors influencing its evolution, and we would only have a poor idea of what damage it would cause if it did. If, for example, the probability of impact somewhere on Earth in 15 years' time were to be estimated at $5 \%$, politicians could argue that with a $95 \%$ probability of a miss there is hardly any justification for action to be taken (depending, perhaps, on whether their part of the world were in the risk corridor!). Indeed, one would normally expect the impact probability to suddenly drop, after initially rising, as further observations reduce the uncertainty region so that Earth is no longer within it (see, e.g., Chesley and Spahr, 2004). However, if Earth were to remain within the uncertainty region as more observational data are gathered, the probability might rise to levels at which an international consensus for action builds, regardless of other circumstances, and valuable time would have been lost for characterizing the object and preparing a spaceborne deflection mission. One of the most difficult challenges faced by astronomers and scientists involved in impact-hazard assessment is ensuring that politicians and decision makers are adequately informed of, and understand, the level of the risk at each stage and how it might develop with time, i.e., how to interpret evolving impact probabilities based on uncertain observational data and incomplete knowledge of factors influencing a NEO's orbit. Critical decisions

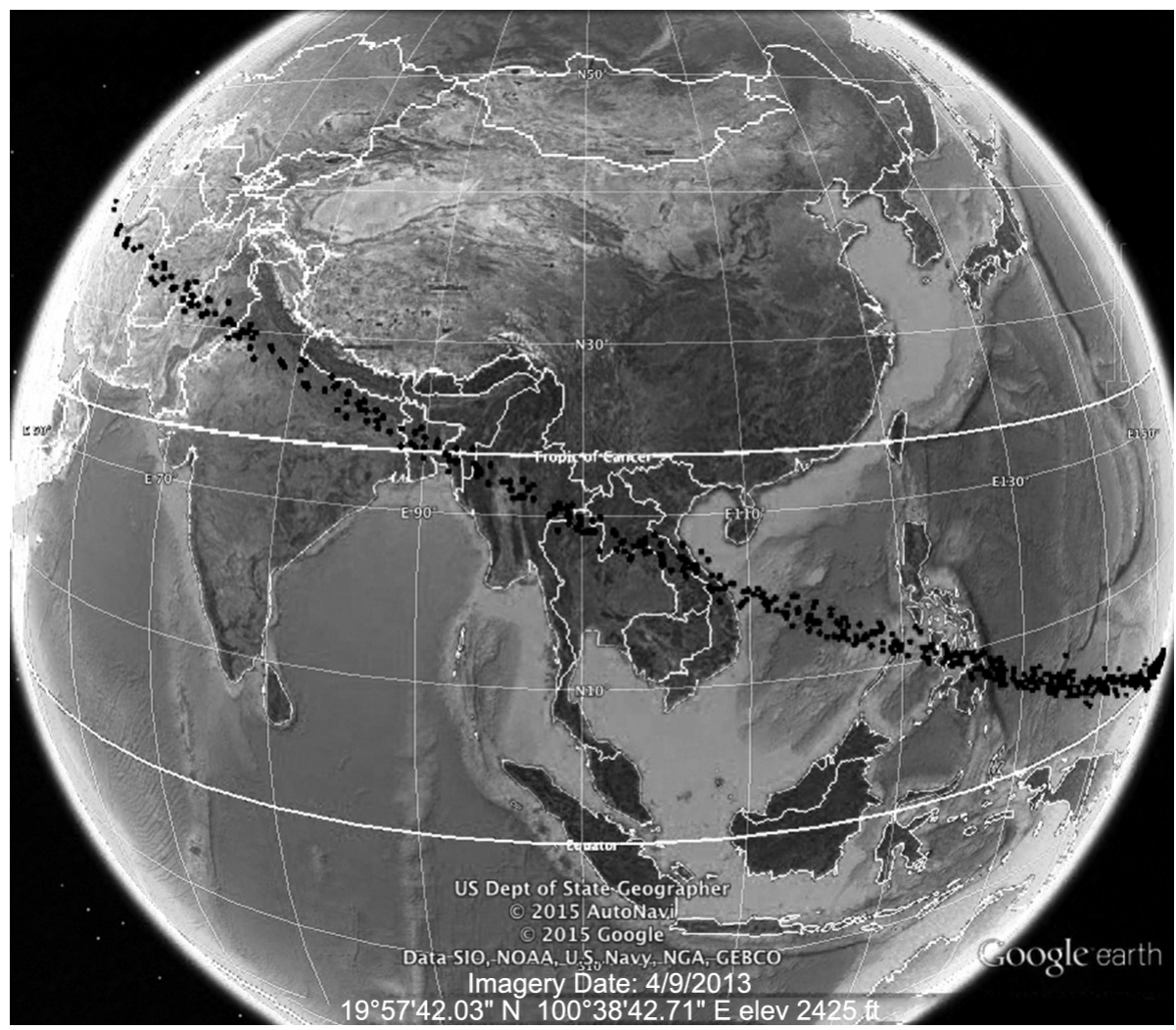

Fig. 3. Example of a hypothetical impact-risk corridor, traced by the black dots extending from the eastern Pacific Ocean, through the Philippines, Southeast Asia, India, to Turkey. In the event the fictional asteroid were to impact Earth, it would do so somewhere within the region traced by the dots. Credit: P. Chodas, NASA/JPL [emergency response exercise conducted during the International Academy of Astronautics (IAA) 2015 Planetary Defense Conference in Frascati, Italy, April 13-17, 2015]. 
would have to be made as a threatening impact scenario evolves concerning what type(s) of mitigation or deflection measure(s) need to be implemented, depending on the size, hence potential destructive power, of the threatening NEO. The answers necessarily depend on some other aspects of the specific scenario, such as duration of advance warning, whether the predicted impact will be in or over an ocean or instead will involve a populated land region, or the availability of heavy-lift launch vehicles.

From a politician's point of view the consequences of "getting it wrong" would be a prime consideration in the decision-making process. Spending the equivalent of hundreds of millions, or billions, of dollars on a deflection campaign, only to learn at a later stage that new observational data imply that the hazardous object will in fact just miss Earth, or that its potential for damage had been grossly overestimated, could lead to serious loss of credibility in the eyes of the public, not only for the politicians involved but also for the scientific community. On the other hand, ignoring a threat that turns out to have more serious consequences than nominally assumed from the available observational data and estimated probabilities would be a far worse outcome. In any case, civil defense planning should always be considered when a threatening object is identified, not only in the case of small objects, e.g., on the scale of Chelyabinsk, but also for larger objects as a back-up measure in case a deflection mission fails. At least in the next couple of decades, it is most likely that predicted impacts by small NEAs will involve days to weeks, or possibly months, of warning, because in general small NEAs would be detected during their final plunges to Earth (see section 5). Such warning times are typical of, or longer than, warning times for evacuation of cities or coastlines in the cases of other natural disasters, such as hurricanes/typhoons. But in most cases, such as those resembling, or smaller than, Chelyabinsk, sheltering in place and staying away from windows is preferable to evacuation. As a precaution against unusual circumstances or early uncertainties about the mass of a recently discovered NEA, such warnings might even be given for predicted impacts by bodies estimated to be as small as a few meters in diameter.

Above what size of impactor would a deflection mission be called for? Since development and execution of such a mission takes years to perhaps a decade, the question of whether or not to seriously embark on such an endeavor will most likely arise well before the magnitude of the threat is well known. In particular, how large an impactor can be allowed to strike without a deflection? And does a spacefaring nation start spending money on such a mission when the probability of striking Earth is just $1 \%$, or must it be $50 \%$ or greater? If the probability of striking Earth were only a few percent, it seems unlikely that society would fund such an ambitious project. Perhaps $10 \%$ or $30 \%$ is a more appropriate threshold for action, although it would certainly depend on the specifics of the threat, and it would be influenced by public attitudes and political and economic considerations. In almost all cases, the risk corridor will be known soon after discovery of the threat, even though the probability of actually impacting may be very low. Assuming, as is probable, that the risk corridor does cross populated regions, it is unlikely (despite the Chelyabinsk example) that it will threaten a major population area or truly critical infrastructure, in which case allowing a Tunguska-sized object to enter the atmosphere, and employing civil defense measures only, should be a reasonable response (unless it is known in advance that the object has a metallic composition; see section 5). It is more problematic to allow a 50-100-m-diameter NEA strike without a deflection attempt, but surely above $100 \mathrm{~m}$ diameter a deflection would seem to be mandatory, if feasible.

In terms of the development of appropriate deflection techniques, we have only just started to consider realistic possibilities; indeed, the choice of deflection technique will depend on knowledge of the physical properties of the threatening object (see, e.g., Michel, 2013). There are many schools of thought on how asteroids could be deflected (section 4). The kinetic impactor appears to offer a realistic approach for objects in the size range 100-500 m. Sending a spacecraft to collide with an asteroid at high relative velocity appears to be feasible, although this method and its associated technology remain to be verified in a test mission. Studies, such as those of the Don Quijote and AIDA concepts (see section 4), imply that the technique could provide an impulse of sufficient magnitude to deflect an object in the aforementioned size range (the secondary of a binary object in the case of AIDA), given some years of warning time, depending on the size and orbit of the asteroid. However, there are many outstanding questions associated with the kinetic impactor technique: How much impactor kinetic energy may be wasted in compaction and restructuring, rather than excavating momentum-enhancing ejecta? How does the target NEA's momentum change depend on parameters such as its bulk density, porosity, mineralogy, and subsurface/ internal structure, and the velocity vector of the impactor relative to the NEA (see the chapter by Jutzi et al. in this volume)? Can ejecta production be characterized in terms of mineralogy and taxonomic type? If so, such knowledge would greatly increase the value of groundbased observations for deflection mission planning. Preliminary work carried out within the European-funded NEOShield project suggests that the momentum transferred by an artificial projectile to a nonporous body is significantly greater (by up to $10 \times$ the momentum of the projectile) than to a porous body (Jutzi and Michel, 2014), a result that is consistent with laboratory impact experiments using scaling laws to extrapolate the results to asteroid scales (e.g., Holsapple and Housen, 2012). However, much work remains to be done to enable reliable predictions to be made as to how a NEA with a particular set of physical parameters would respond to an impacting spacecraft.

Furthermore, there are a number of important issues relating to the targeting accuracy achievable with current technology. The efficiency of momentum transfer from the impactor to the hazardous NEA depends crucially on the impact accuracy. While linear momentum is conserved, a 
spacecraft impacting near the end of an elongated object would waste kinetic energy in changing the spin state of the object rather than producing momentum-enhancing ejecta. The most effective collision trajectory would be aimed at the center of mass of the asteroid. How would the necessary camera resolution and autonomous control-loop response time depend on the approach velocity, target size, thruster sizing, and required accuracy of the impact location? What is the trade-off between impactor approach trajectory, impact accuracy, and potentially unfavorable illumination conditions (e.g., approach from a high solar phase angle)? These are examples of questions that need to be addressed before we could be confident that a kinetic-impactor mission would have the desired effect. It is clear from the above discussion that not only does the kinetic impactor technique have an upper size limit on its applicability due to current limitations on launch mass, but also a lower size limit given by the targeting accuracy achievable with current autonomous guidance, navigation, and control systems.

Other currently favored deflection techniques that may be more suited to smaller bodies, or cases in which only a small deflection is required (such as avoidance of a "keyhole" see section 3), include the so-called slow-push (or -pull) techniques, such as those involving the use of lasers or ion beams and the gravity tractor (see section 4 ). Such techniques differ considerably in terms of their maturity. The gravity tractor concept has the advantage of relative simplicity and, given current technology (but as yet without the benefit of practical experience), is considered to be the most reliable option in the class of non-impulsive techniques.

If the discovery of a threatening NEA leads to the decision to develop a deflection mission, which kind of deflection technique would be most appropriate? Here we assume currently available launch vehicle capabilities, a few decades of warning, a NEA bulk density of $2 \mathrm{~g} \mathrm{~cm}^{-3}$, and that populated regions are near ground zero or near the risk corridor. We assume that the purpose of deflection is to miss Earth by a comfortable margin ( $2.5 \mathrm{R}_{\oplus}$ from Earth's center), although there will be cases where the much easier task of deflection - to miss a small keyhole - is all that is required. These issues were evaluated by Shapiro et al. (2010). It is anticipated that one or a series of kinetic impactors could reasonably deflect an NEA of $500 \mathrm{~m}$ to $\leq 1 \mathrm{~km}$ in diameter. For most scenarios involving larger bodies, a nuclear device is the only sufficiently energetic approach for deflection. A gravity tractor acting for a decade could deflect a NEA of up to $\sim 50 \mathrm{~m}$ in diameter. So the kinetic impactor approach to deflection would be appropriate for most of the truly dangerous scenarios for which deflection is mandatory in order to prevent a regional catastrophe (e.g., an impact that could lay waste to land areas the size of a country or a U.S. state). Such an approach, for NEAs mainly in the diameter range of 100 to $500 \mathrm{~m}$, would necessarily require a high degree of reliability, which could be assured by appropriate physical characterization of the threatening body by precursor investigation, supplemented by an observer spacecraft to witness the deflection operation and assess its success, and finally, if necessary, function as a gravity tractor. It would, of course, be desirable to have backup kinetic impactors and gravity tractors in case the first ones fail.

One can question whether a deflection technique with excessive capability might be used on a smaller NEA. For example, a nuclear device could surely deflect or even destroy a NEA much smaller than $0.5-1 \mathrm{~km}$. We suggest that such possible use is not realistic because of the serious ancillary political issues involving development of such a capability, legal issues concerning practicing such a capability in outer space, and expected widespread public objection to using such a technique, except perhaps as a measure of last resort. On the other hand, there is the concern that procrastination in approving and implementing feasible nonnuclear deflection missions might leave nuclear as the only last-minute option, which would pose a serious geopolitical dilemma.

Should kinetic impactors be used even for NEAs smaller than $100 \mathrm{~m}$, where a slow-push/pull method could be sufficient? Here, the answer may be "yes." Of course, the kinetic impactor approach is inherently less precise because it involves direct interaction with the possibly heterogeneous surface of the NEA for which the momentum enhancement factor may be very uncertain. However, a kinetic impactor can accomplish its task with an instantaneous strike rather than having to operate reliably for many years.

In Table 1 we give an overview of our current state of knowledge (or perhaps ignorance!) of the impact hazard for different sizes of impactor and appropriate mitigation strategies given current technology. Some knowledge of the physical properties of a threatening NEA is necessary to ensure the successful outcome of a deflection attempt. The type of knowledge required depends on the deflection technique in question (see, e.g., Michel, 2013): A gravity tractor has to operate for long periods of time in close vicinity to the NEA, but does not physically interact with the surface; in this case, therefore, accurate information on the mass, shape, and rotation vector of the object are required, which can be obtained by the spacecraft after it arrives at the object, but other physical properties are largely irrelevant. On the other hand, in the case of a kinetic impactor, knowledge of density, porosity, and internal structure would also be important for a predictable outcome. In the case of a deflection mission based on a laser, ion-beam, or explosive device, knowledge of the mineralogical composition of the surface material would also be relevant.

The NEOShield consortium, among others, has performed studies of observational data on NEAs (see, e.g., Harris and Drube, 2014) and laboratory experiments, including hypervelocity impacts on asteroid surface analog materials, and carried out related computer simulations (see, e.g., Jutzi and Michel, 2014). While our knowledge of NEA properties has grown significantly over the past decade, our knowledge of the deflection-relevant internal structures of NEAs is still seriously lacking. Available data on the distribution of lightcurve amplitudes and spin rates (Fig. 4) suggest that many fast-spinning objects take on more symmetrical shapes, presumably due to restructuring caused by spin-induced movement and relocation 
TABLE 1. Estimated near-Earth asteroid numbers, impact intervals, impact consequences, and mitigation possibilities.

\begin{tabular}{|c|c|c|c|c|}
\hline $\begin{array}{l}\text { NEA } \\
\text { Diameter, } \\
\text { D (m) }\end{array}$ & $\begin{array}{l}\text { Estimated Total } \\
\text { Number* with } \\
\text { Diameter } \geq \mathrm{D} \text { in the } \\
\text { NEA Population }\end{array}$ & $\begin{array}{l}\text { Indicative Impact } \\
\text { Interval }(\mathrm{yr})^{*} \text { for } \\
\text { NEAs with } \\
\text { Diameter } \geq \mathrm{D}\end{array}$ & $\begin{array}{l}\text { Possible Consequences of Impact } \\
\text { Near Populated Region }{ }^{\dagger}\end{array}$ & $\begin{array}{c}\text { Appropriate Mitigation/Deflection } \\
\text { Strategy Assuming Current } \\
\text { Technology }\end{array}$ \\
\hline 10 & 100 million & 5 & Meteorite falls; crater unlikely & Civil defense only \\
\hline 30 & 3 million & 150 & $\begin{array}{l}\text { Chelyabinsk/Tunguska-type } \\
\text { airburst; crater, depending on } \\
\text { composition; some injuries and } \\
\text { deaths }\end{array}$ & Civil defense only \\
\hline 50 & 500,000 & 1000 & $\begin{array}{l}\text { Violent Tunguska-type airburst; } \\
\text { crater, depending on composition; } \\
\text { potentially many injuries and } \\
\text { deaths }\end{array}$ & $\begin{array}{l}\text { Slow push/pull (e.g., gravity } \\
\text { tractor) or kinetic impactor, if } \\
\text { feasible, civil defense only if not }\end{array}$ \\
\hline 100 & 50,000 & 10,000 & $\begin{array}{l}\text { Crater } 1-2 \mathrm{~km} \text { in diameter; local } \\
\text { destruction; tsunami risk from near- } \\
\text { shore impacts; many deaths likely }\end{array}$ & $\begin{array}{l}\text { Civil defense; slow push/pull or } \\
\text { kinetic impactor — slow push/ } \\
\text { pull combination }\end{array}$ \\
\hline 300 & 7000 & 70,000 & $\begin{array}{l}\text { Crater several kilometers in } \\
\text { diameter; regional/national } \\
\text { destruction; tsunami risk; } \\
\text { potentially millions of deaths }\end{array}$ & $\begin{array}{l}\text { Kinetic impactor - slow push/ } \\
\text { pull combination if feasible, } \\
\text { explosive impulse if not, plus } \\
\text { civil defense if practical }\end{array}$ \\
\hline 500 & 3500 & 140,000 & $\begin{array}{l}\text { Crater some } 10 \mathrm{~km} \text { in diameter; } \\
\text { international catastrophe; tsunami } \\
\text { risk; potentially millions of deaths }\end{array}$ & $\begin{array}{l}\text { Kinetic impactor - slow push/ } \\
\text { pull combination if feasible; } \\
\text { several kinetic impactors may be } \\
\text { necessary. Explosive impulse if } \\
\text { no alternative }\end{array}$ \\
\hline 1000 & 1000 & 500,000 & $\begin{array}{l}\text { Global effects; partial disruption of } \\
\text { civilization }\end{array}$ & $\begin{array}{l}\text { Series of kinetic impactors or } \\
\text { large explosive impulse }\end{array}$ \\
\hline 10,000 & $3 *$ & 100 million & End of present civilization & $\begin{array}{l}\text { Series of large explosive } \\
\text { impulses; deflection may not be } \\
\text { feasible with current technology }\end{array}$ \\
\hline
\end{tabular}

of material in a rubble-pile-like structure with little cohesion. The fact that binary objects cluster just below the spin barrier for gravity-dominated bodies with diameters larger than about $300 \mathrm{~m}$ (spin period $\sim 2 \mathrm{~h}$ ) also suggests that many objects in this size category have insufficient cohesion or strength to withstand faster rotation (Figs. 4 and 5); on the other hand, many of the asteroids smaller than $300 \mathrm{~m}$ rotate much faster, implying they must have a cohesive structure, even if just a small amount of cohesion appears to be sufficient for them to survive (Holsapple, 2007). Sánchez and Scheeres (2014) hypothesize that van der Waals forces acting between small dust grains in a rubble pile can give rise to cohesion: Boulders and pebbles of diverse sizes could be held in place by a surrounding matrix of finer material. In this picture even very small objects with large lightcurve amplitudes (and therefore probably elongated shapes) could exist as rubble piles but spin more rapidly than the spin barrier for gravity-dominated objects. For example, work by Rozitis et al. (2014) implies that the fast-spinning kilometer-sized NEA (29075) 1950 DA is a rubble pile held together by weak cohesive forces comparable to, or less than, the forces between grains in lunar regolith. However, if the spin rate of a rubble pile is increased, as a result of the Yarkovsky-O'Keefe-Radzievskii-Paddack (YORP) effect or a close planetary encounter, then the object could disintegrate, giving rise to small monolithic bodies. While observational data and modeling results are consistent with rubble-pile structures being common among NEAs, the fact that numerous small objects spin at rates above the Sánchez and Scheeres (2014) disruption limit (Fig. 5) suggests the possible existence of monolithic asteroids at the small end of the size distribution.

In any case, the fact that small asteroids can have very high rotation rates (some measurements exceed $1 \mathrm{rev} / \mathrm{min}$ ) is an important consideration for a deflection mission. In the case of a kinetic impactor, fast rotation, combined with imperfect knowledge of the target's shape, and rapid approach from a 
high solar phase angle could significantly increase the risk of a navigation error and failure to impact at the required position. In the case of a gravity tractor, maneuvering and station keeping close to the surface of an irregularly shaped

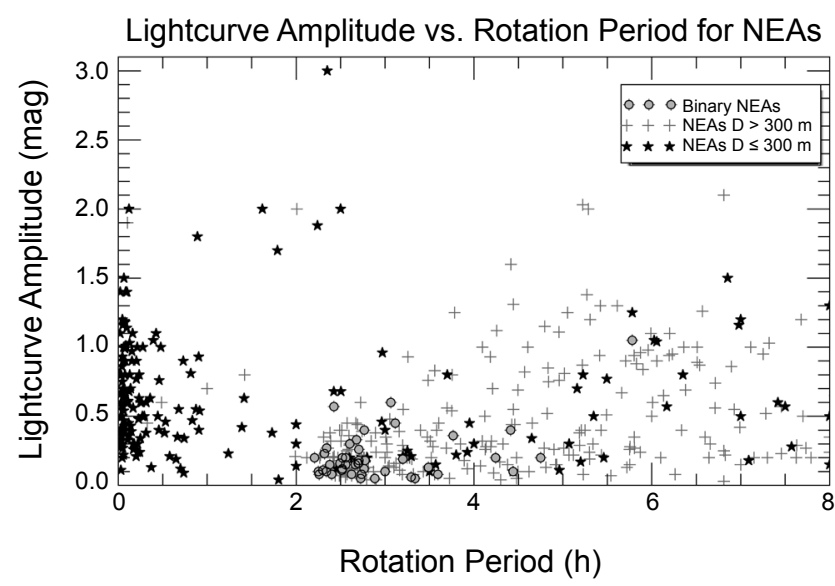

Fig. 4. Lightcurve amplitude vs. spin period for NEAs. Data are from the European Asteroid Research Node (EARN) database (http://earn.dlr.de/). The data suggest that many fast-spinning objects with diameters larger than $300 \mathrm{~m}$ take on more symmetrical shapes (and thus have smaller lightcurve amplitudes), presumably due to restructuring caused by spin-induced movement and relocation of material in a rubble-pile-like structure with little cohesion. On the other hand, many of the asteroids smaller than $300 \mathrm{~m}$ rotate much faster, implying they must have a somewhat cohesive structure.

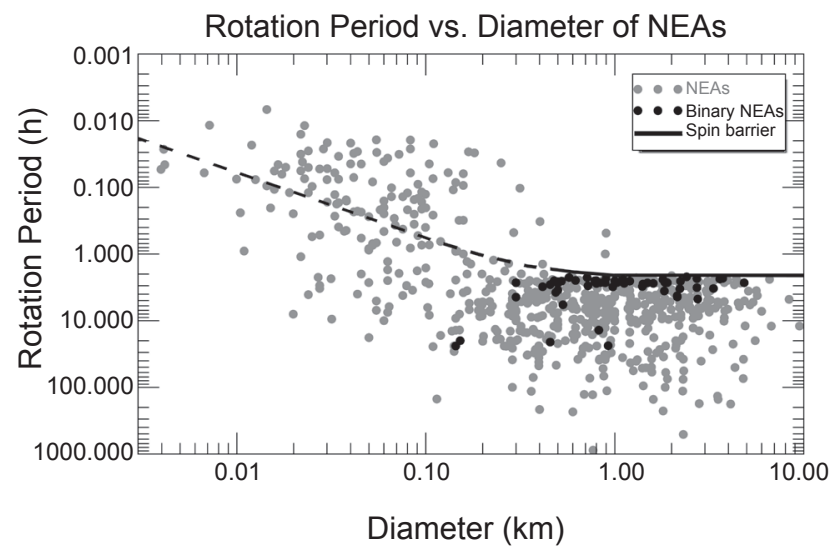

Fig. 5. Rotation period vs. size for NEAs. Data are from the EARN database (http://earn.dlr.de/). The "spin barrier" for gravity-dominated bodies (spin period $\sim 2 \mathrm{~h}$, diameters larger than about $300 \mathrm{~m}$ ) is shown merging into the disruption limit (dashed line) of Sánchez and Scheeres (2014) for smaller bodies. The plotted disruption limit corresponds to a cohesion strength of $25 \mathrm{~Pa}$, which is consistent with available data according to Sánchez and Scheeres (2014). It should be noted, however, that a higher strength is required to enclose the super-fast rotators among smaller (<100-mdiameter) objects. Thus, the internal strength of many smaller NEOs must be greater than that of the larger ones. asteroid with an irregular gravity field become more difficult at high rotation rates. Since fast-rotating NEAs appear to be common, and deflecting a fast-rotating target is likely to be technically challenging, a deflection demonstration mission targeting a representative relatively fast rotator would provide a revealing test of current NEA deflection capabilities.

Studies of NEA physical properties, including laboratory work, numerical modeling, and computer simulations, are essential aspects of a program to develop deflection technology, but they are not sufficient to enable robust predictions to be made of the outcome of a deflection attempt. We may one day be faced with having to decide on a risky deflection mission without having time to acquire adequate information on the physical properties of the threatening object. We would obviously obtain the best knowledge of the body possible using available astronomical facilities, but that might still result in uncertainties of a factor of a few in the body's mass and ignorance of other relevant characteristics. A "bang and hope" mission with an uncertain outcome would be our only option, although we could improve our chances by preparing back-up missions and taking civil defense action. In any case, to minimize the uncertainties in the outcome of a deflection mission, it would seem prudent to test our concepts and assumptions in experimental missions to deflect suitable representative NEA targets before we are forced to apply deflection technology in a hurry in a real impact-prevention scenario.

\section{INTERNATIONAL COORDINATION EFFORTS AND THE POLITICAL DIMENSION}

Protecting Earth from NEO impacts is a global problem and, as such, any deflection strategy should involve at least the most scientifically and technologically capable nations. However, all nations, especially those with large geographical areas or regions of high population density, should be encouraged to take part in discussions and decision-making concerning mitigation planning in general. Impact-hazard-related activities at the UN were prompted by the recommendations of the Third United Nations Conference on the Exploration and Peaceful Uses of Outer Space (UNISPACE III) held in Vienna in July 1999. Following the UNISPACE III recommendations the Action Team on Near-Earth Objects (AT-14) was established by the UN's Committee on the Peaceful Uses of Outer Space (COPUOS), an intergovernmental body set up in 1959 to "encourage continued research and the dissemination of information on outer space matters and to study legal problems arising from the exploration of outer space." Action Team 14, which is composed of both government and nongovernment experts, was charged with reviewing the ongoing efforts in the field of NEOs, identifying fields in which further effort is required, or where other countries/ organizations could contribute, and proposing steps for the improvement of international coordination. The work of AT-14 continued until 2008 when the team presented a summary of the recommendations contained in the report Asteroid Threats: A Call for Global Response, prepared by the Association of Space Explorers (ASE) (http://www.space-explorers.org). 
To accelerate the formal processing of the ASE report within the UN, the Scientific and Technical Subcommittee of COPUOS established a working group at the government level for the purposes of formally receiving and assessing the recommendations of the report. Subsequently, in the light of the ASE/AT-14 recommendations, the focus of AT-14 changed to reviewing policies and proposing procedures for handling the NEO threat on the international stage. As a direct result of the work of AT-14 and the ASE, the establishment of two new groups (Fig. 6) was endorsed by the sixty-eighth session of the UN General Assembly in December 2013: the IAWN and the SMPAG. The purpose of the IAWN is to link together institutions carrying out discovery, monitoring, and physical characterization of potentially hazardous NEOs with other relevant organizations, and promote an internationally recognized clearinghouse to manage NEO observations and impact predictions. While some of these functions are already covered by existing bodies, such as the Minor Planet Center at the Smithsonian Astrophysical Observatory, which operates under the auspices of the International Astronomical Union, the IAWN is intended to enhance cooperation between relevant organizations and to be the UN-mandated international portal for accurate and validated information on the NEO population, as well as to provide recommendations for communication strategies for governments and other relevant bodies in the event of an emerging impact threat. NASA has agreed to be the initial coordinator of the IAWN.
The IAWN is also charged with establishing collaboration with international disaster response agencies in order to develop emergency plans in response to an impact threat in a populated area. NASA sponsored a tabletop exercise in April 2013 to simulate an impact on Earth for the U.S. Federal Emergency Management Agency (FEMA). A further impact simulation exercise was held during the Planetary Defense Conference in Flagstaff, Arizona, also in 2013 (Carnelli et al., 2014), at which FEMA participated. A scenario in which the probability of impact of a 200-300$m$ NEA increased over a period of 15 years was simulated. More than 200 exercise participants were allocated to groups to take charge of different aspects of mitigation planning (e.g., NEO discovery and follow-up, mitigation techniques and missions, impact effects, media and risk communication, launch capability of space agencies, etc.). FEMA and NASA have recently established a NEO Impact Working Group, to be responsible for reviewing disaster response and recommend future exercises. In 2014, ESA also held an information meeting and similar tabletop exercise for representatives of the European emergency response community. Further information meetings involving emergency coordination agencies, including those of the $\mathrm{UN}$, are planned and will help to establish a dialog between the scientific and technical impact hazard community and those that would be responsible for disaster management in the event of an impact emergency.

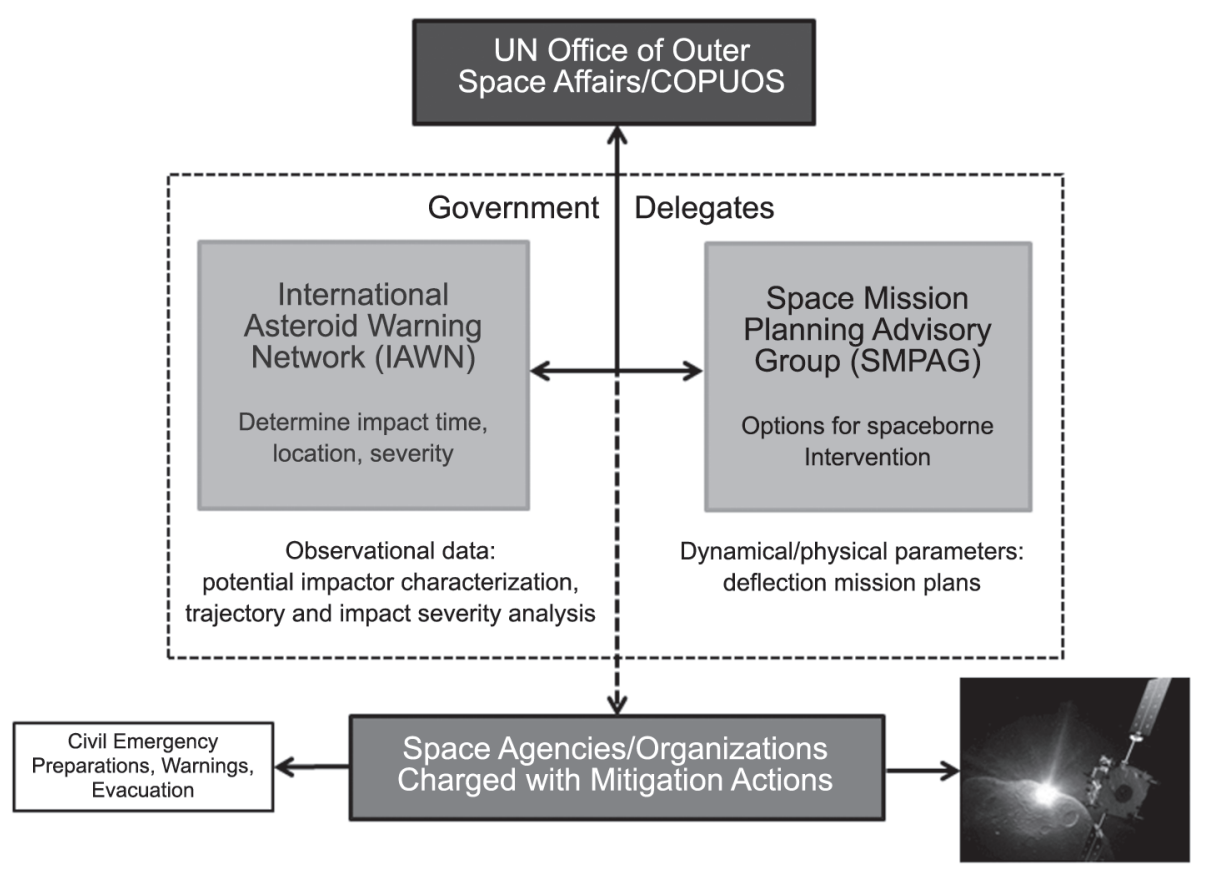

Fig. 6. Draft scheme of the organization of NEO impact hazard activities under the auspices of the United Nations. Details of the tasks, responsibilities, and funding of the IAWN and SMPAG, and the assignment of mandates to take action in an emergency situation, are under discussion at the present time. The vertical dashed arrow represents channels that have yet to be formally established. Contacts with civil defense organizations are currently being developed. Present membership of the SMPAG includes space agencies that would be capable of contributing to a spaceborne deflection attempt. Kinetic impactor illustration courtesy of ESA-AOES Medialab. 
The SMPAG was established to promote opportunities for international collaboration on research and development work relating to NEO deflection and to develop a framework, timeline, and options for initiating and executing spacemission-response activities. The SMPAG should also develop a set of reference missions, addressing a variety of potential NEO impact scenarios and deflection/disruption possibilities, to facilitate realistic technical and resource planning. Whenever a credible impact threat is established by the IAWN, the SMPAG is charged with proposing options for spaceborne intervention to divert the threatening object. Membership in the SMPAG is open to all national space agencies or governmental or intergovernmental entities that coordinate and fund space activities and are capable of carrying out, or contributing to, a spaceborne NEO deflection campaign. The first chair of the SMPAG is ESA; the chairmanship will rotate around the members at intervals of two years.

Information on the history, development, and status of UN activities relating to NEOs and the impact threat is available from http://www.oosa.unvienna.org/oosa/en/COPUOS/ stsc/wgneo/index.html.

The IAWN and SMPAG are asked to work together on recommendations for criteria and thresholds for mitigation action, such as notification of a significant impact risk or the initiation of observation/mitigation campaigns. Both groups are currently still in their infancy and questions regarding such details as their ultimate tasks and responsibilities, schedule of activities, etc., are still open at the time of this writing. A pressing current concern with regard to the initiatives of the $\mathrm{UN}$ is financial provision for the tasks involved. The work of both the IAWN and SMPAG has to be carried out at no cost to the UN budget. Consequently, the states and organizations making up the membership of the two UN groups have to fund their involvement from their own resources. A model for the support of the activities of the groups might be the current funding of the NEOShield project by the European Commission. While 11 of NEOShield's 13 partner organizations are from European Union countries, two are from non-EU countries, namely Russia and the U.S., but they also receive funding from the European Union. Therefore NEOShield is a truly international project. Taking the SMPAG, for example, statements or "white papers" on future research and development requirements in the field could be issued by the SMPAG on a regular basis, carrying the authority of the UN. Calls for proposals from funding agencies, such as the European Commission or national agencies, could refer to the SMPAG statements, and proposers could orient their work plans accordingly. A plausible vision for the future would be coordinated calls for mitigation-related proposals from a combination of international and national funding bodies, based on the recommendations of the UN-backed groups.

\section{SUMMARY AND CONCLUSIONS}

The period since the publication of Asteroids III has seen rapid progress in many fields related to impact hazards. Optical search programs benefiting from advances in detector technology and sophisticated automated detection routines are currently enjoying increased funding from NASA. The rate of discovery of NEOs has increased to an average of some 3 per day and the number of NEOs discovered of all sizes has risen from around 2000 at the end of 2002 to some 11,000 at the time of this writing. While endeavors in the field of physical characterization have not kept up with the rate of new discoveries, the NEOWISE and ExploreNEO projects have provided size and albedo data for many hundreds of NEOs and demonstrated the value of spacebased telescopes sensitive in the thermal infrared for NEO risk assessment.

Unfortunately, the unanticipated Chelyabinsk event on February 15, 2013, has demonstrated that the blast waves from even relatively small (diameter $\sim 20 \mathrm{~m}$ ) asteroids exploding in the atmosphere can cause serious injuries and damage to property. Chelyabinsk has also taught us that a potentially serious impact can occur with no prior warning if the orbit of the NEO happens to be unfavorable for detection from the ground.

A number of initiatives suggest that the NEO impact threat is attracting increasing attention in political circles not only in the U.S. The European Commission funded the NEOShield project from 2012 to 2015, enabling 13 academic and industrial organizations from 6 countries, including the U.S. and Russia, to collaborate on investigating NEO physical characteristics and deflection options. European activities also include the NEO segment of ESA's Space Situational Awareness program, which is currently under development and includes plans to build the "Fly-Eye" telescope system with the aim of providing sufficient warning of small impactors to allow civil defense measures to be taken. A U.S. project with similar aims is the ATLAS system. New groups have been established under the auspices of the UN to help to coordinate astronomical NEO survey and characterization activities (IAWN) and to advise on deflection-related space mission planning (SMPAG). The UN initiatives demonstrate that concern about the NEO impact hazard is increasing worldwide.

Despite the encouraging progress made during the past decade or so, there is still much to be done before we can be confident of our ability to deflect a threatening NEO. Evidence from observations, laboratory impact experiments, and theoretical work suggest that NEOs differ widely in the nature of their near- surface structure, and the outcome of impulsive techniques, such as the kinetic impactor, would be difficult to predict in the absence of information on bulk density, porosity, tensile strength, etc. While alternative slow-push or -pull techniques, such as the gravity tractor, may be suitable in certain circumstances, such as keyhole avoidance, it seems at present that the most likely scenario in which a deflection mission is called for would involve an impulsive technique, perhaps together with a combination observer/gravity tractor spacecraft to provide reconnaissance data and the opportunity to adjust the post-impulse trajectory of the target.

While studies of a number of deflection techniques have been carried out, no technique has yet been tested in space on a real NEA. In order to minimize the uncertainties in the outcome of a deflection mission, it would certainly be 
prudent to test our concepts and assumptions in experimental missions to deflect suitable representative NEA targets before we are forced to apply deflection technology in a hurry in a real impact-prevention scenario.

Acknowledgments. We thank L. Johnson, Program Executive for NASA's NEO Observations Program, for information on the status of NASA NEO initiatives. We thank our referees, D. Morrison and D. Yeomans, for their thorough reviews, which have led to significant improvements in the presentation of this work. Some of the research described in this article has received funding from the European Union's Seventh Framework Program (FP7/2007-2013) under grant agreement No. 282703 (NEOShield).

\section{REFERENCES}

A’Hearn M. F., Belton M. J. S., Delamere W. A., et al. (2005) Deep impact: Excavating comet Tempel 1. Science, 310, 258-264, DOI: 10.1126/science. 1118923 .

Belton M. J. S., Morgan T. H., Samarasinha N., and Yeomans D. K., eds. (2004) Mitigation of Hazardous Comets and Asteroids. Cambridge Univ., Cambridge. 414 pp.

Binzel R. P., Lupishko D. F., Di Martino M., Whiteley R. J., and Hahn G. (2002) Physical properties of near-Earth objects. In Asteroids III (W. F. Bottke Jr. et al., eds.), pp. 255-271. Univ. of Arizona, Tucson.

Birks J. W., Crutzen P. J., and Roble R. G. (2007) Frequent ozone depletion resulting from impacts of asteroids and comets. In Comet/Asteroid Impacts and Human Society (P. Bobrowsky and H. Rickman, eds.), pp. 225-245. Springer-Verlag, Berlin.

Bobrowsky P. and Rickman H., eds. (2007) Comet/Asteroid Impacts and Human Society. Springer-Verlag, Berlin. 546 pp.

Bombardelli C., Urrutxua H., Merino M., Peláez J., and Ahedo E. (2013) The ion beam shepherd: A new concept for asteroid deflection. Acta Astronaut., 90, 98-102, DOI: 10.1016/j.actaastro.2012.10.019.

Boslough M. (2014) Airburst warning and response. Acta Astronaut., 103, 370-375, DOI: 10.1016/j.actaastro.2013.09.007.

Boslough M. B. E. and Crawford D. A. (1997) Shoemaker-Levy 9 and plume forming collisions on Earth. In Near-Earth Objects (J. L. Remo, ed.), pp. 236-282. Annals of the New York Academy of Sciences, Vol. 822, New York, DOI: 10.1111/j.1749-6632.1997.tb48345.x.

Boslough M. B. E. and Crawford D. A. (2008) Low altitude airbursts and the impact threat. Intl. J. Impact Eng., 35, 1441-1448, DOI: 10.1016/j.ijimpeng.2008.07.053.

Brown P., Spalding R. E., ReVelle D. O., Tagliaferri E., and Worden S. P. (2002) The flux of small near-Earth objects colliding with the Earth. Nature, 420, 294-296, DOI: 10.1038/nature01238.

Brown P. G., Assink J. D., Astiz L. et al. (2013) A 500-kiloton airburst over Chelyabinsk and an enhanced hazard from small impactors. Nature, 503, 238-241, DOI: 10.1038/nature12741.

Buratti B. J. and Johnson L. L. (2003) Identification of the lunar flash of 1953 with a fresh crater on the Moon's surface. Icarus, 161, 192-197, DOI: 10.1016/S0019-1035(02)00027-1.

Carnelli I., Ailor W., and Tremayne-Smith R. (2014) NEO Planetary Defense 2013: Gathering for impact. Acta Astronaut., 103, 307-308, DOI:10.1016/j.actaastro.2014.08.004.

Chapman C. and Morrison D. (1994) Impacts on the Earth by asteroids and comets: Assessing the hazard. Nature, 367, 33-40, DOI: $10.1038 / 367033 \mathrm{a} 0$

Cheng A. F. (2002) Near Earth Asteroid Rendezvous: Mission summary. In Asteroids III (W. F. Bottke Jr. et al., eds.), pp. 351-366. Univ. of Arizona, Tucson.

Cheng A. F., Atchison J., Kantsiper B., Rivkin A. S., Stickle A., Reed C., Galvez A., Carnelli I., Michel P., and Ulamec S. (2015) Asteroid Impact and Deflection Mission. Acta Astronaut., 115, 262-269, DOI: 10.1016/j.actaastro.2015.05.021.

Chesley S. R. and Spahr T. B. (2004) Earth impactors: Orbits and warning times. In Mitigation of Hazardous Comets and Asteroids (M. J. S. Belton et al., eds.), pp. 22-37. Cambridge Univ., Cambridge

Collins G. S., Melosh H. J., and Marcus R. A. (2005) Earth impact effects program: A web-based computer program for calculating the regional environmental consequences of a meteoroid impact on
Earth. Meteoritics \& Planet. Sci., 40, 817-840, DOI: 10.1111/j.19455100.2005.tb00157.x.

Farnocchia D., Bernardi F., and Valsecchi G. B. (2012) Efficiency of a wide-area survey in achieving short- and long-term warning for small impactors. Icarus, 219, 41-47, DOI: 10.1016/j.icarus. 2012.02.014.

Farnocchia D., Chesley S. R., Chodas P. W., Tricarico P., Kelley M. S. P., and Farnham T. L. (2014) Trajectory analysis for the nucleus and dust of Comet C/2013 A1 (Siding Spring). Astrophys. J., 790, 114, DOI: 10.1088/0004-637X/790/2/114.

Foster C., Bellerose J., Mauro D., and Jaroux B. (2013) Mission concepts and operations for asteroid mitigation involving multiple gravity tractors. Acta Astronaut., 90, 112-118, DOI: 10.1016/ j.actaastro.2012.10.010.

Fujiwara A., Kawaguchi J., Yeomans D. K., et al. (2006) The rubble-pile asteroid Itokawa as observed by Hayabusa. Science, 312, 1330-1334, DOI: 10.1126/science.1125841.

Garbolino E. and Michel P. (2011) Proposal of a spatial decision support system architecture to estimate the consequences and costs of small meteorites impacts. Nat. Hazards Earth Syst. Sci., 11, 3013-3021, DOI: 10.5194/nhess-11-3013-2011.

Garshnek V., Morrison D., and Burkle F. M. (2000) The mitigation, management, and survivability of asteroid/comet impact with the Earth. Space Policy, 16, 213-222, DOI :10.1016/S0265-9646(00)00025-4.

Gehrels T., ed. (1994) Hazards Due to Comets and Asteroids. Univ. of Arizona, Tucson. 1300 pp.

Gibbings A., Vasile M., Watson I., Hopkins J.-M., and Burns D. (2013) Experimental analysis of laser ablated plumes for asteroid deflection and exploitation. Acta Astronaut., 90, 85-97, DOI: 10.1016/ j.actaastro.2012.07.008.

Gisler G., Weaver R., and Gittings M. (2011) Calculations of asteroid impacts into deep and shallow water. Pure Appl. Geophys., 168, 1187-1198, DOI: 10.1007/s00024-010-0225-7.

Harris A. W. (2014) Near-Earth-object survey progress and population of small near-Earth asteroids. In Asteroids, Comets, Meteors 2014, Book of Abstracts (K. Muinonen et al., eds.), Helsinki, Finland. Available online at http://www.helsinki.fi/acm2014/pdf-material/ Day-2/Session-3/Room-3/HARRIS-6E69.pdf.

Harris A. W. and Drube L. (2014) How to find metal-rich asteroids. Astrophys. J. Lett., 785, L4, DOI: 10.1088/2041-8205/785/1/L4.

Harris A. W., Barucci M. A., Cano J. L., Fitzsimmons A., Fulchignoni M., Green S. F., Hestroffer D., Lappas V., Lork W., Michel P., Morrison D., Payson D., and Schäfer F. (2013) The European Union funded NEOShield project: A global approach to near-Earth object impact threat mitigation. Acta Astronaut., 90, 80-84, DOI: 10.1016/ j.actaastro.2012.08.026.

Holsapple K. A. (2007) Spin limits of solar system bodies: From the small fast-rotators to 2003 EL61. Icarus, 187, 500-509, DOI: 10.1016/j.icarus.2006.08.012.

Holsapple K. A. and Housen K. R. (2012) Momentum transfer in asteroid impacts. I. Theory and scaling. Icarus, 221, 875-887, DOI: 10.1016/j.icarus.2012.09.022.

Huang J., Ji J., Ye P., et al. (2013) The ginger-shaped asteroid 4179 Toutatis: New observations from a successful flyby of Chang'e-2. Scientific Reports, 3, 3411, DOI: 10.1038/srep03411.

Jutzi M. and Michel P. (2014) Hypervelocity impacts on asteroids and momentum transfer: I. Numerical simulations using porous targets. Icarus, 229, 247-253, DOI: 10.1016/j.icarus.2013.11.020.

Kenkmann T., Artemieva N. A., Wünnemann K., Poelchau M. H., Elbeshausen D., and Núñez Del Prado H. (2009) The Carancas meteorite impact crater Peru: Geologic surveying and modeling of crater formation and atmospheric passage. Meteoritics \& Planet. Sci., 44, 985-1000, DOI: 10.1111/j.1945-5100.2009.tb00783.x.

Korycansky D. G. and Lynett P. J. (2005) Offshore breaking of impact tsunami: The Van Dorn effect revisited. Geophys. Res. Lett., 32, L10608, DOI: 10.1029/2004GL021918.

Kring D. and Boslough M. (2014) Chelyabinsk: Portrait of an asteroid airburst. Phys. Today, 67, 32-37, DOI: 10.1063/PT.3.2515.

Lauretta D. S. and the OSIRIS-REx Team (2012) An overview of the OSIRIS-REx asteroid sample return mission. Lunar Planet. Sci. XLIII, Abstract \#2491. Lunar and Planetary Institute, Houston.

Lu E. T. and Love S. G. (2005) Gravitational tractor for towing asteroids. Nature, 438, 177-178. DOI: 10.1038/438177a.

Mainzer A., Grav T., Bauer J., et al. (2011) NEOWISE observations of near-Earth objects: Preliminary results. Astrophys. J., 743, 156, DOI: $10.1088 / 0004-637 \mathrm{X} / 743 / 2 / 156$. 
Malin M. C., Edgett K. S., Posiolova L. V., McColley S. M., and Noe Dobrea E. Z. (2006) Present-day impact cratering rate and contemporary gully activity on Mars. Science, 314, 1573-1577, DOI: $10.1126 /$ science. 1135156.

Michel P. (2013) Physical properties of near-Earth objects that inform mitigation. Acta Astronaut., 90, 6-13, DOI: 10.1016/j.actaastro. 2012.07.022.

Michel P., Cheng A. F., Küppers M., and the AIDA Team (2015) Asteroid Impact and Deflection Assessment (AIDA) mission: Science investigation of a binary system and mitigation test. EPSC Abstracts, 10, EPSC2015-123, available online at http:// meetingorganizer.copernicus.org/EPSC2015/EPSC2015-123.pdf.

Morrison D., Harris A. W., Sommer G., Chapman C. R., and Carusi A. (2002) Dealing with the impact hazard. In Asteroids III (W. F. Bottke Jr. et al., eds.), pp. 739-754. Univ. of Arizona, Tucson.

Nakamura T., Noguchi T., Tanaka M., et al. (2011) Itokawa dust particles: A direct link between S-type asteroids and ordinary chondrites. Science, 333, 1113-1116, DOI: 10.1126/science.1207758.

Noll K., Weaver H., and Feldman P., eds. (1996) The Collision of Comet Shoemaker-Levy 9 and Jupiter. IAU Colloq. 156, Cambridge Univ., Cambridge.

Ostro S. J. and Giorgini J. D. (2004) The role of radar in predicting and preventing asteroid and comet collisions with Earth. In Mitigation of Hazardous Comets and Asteroids (M. J. S. Belton et al., eds.), pp. 38-65. Cambridge Univ., Cambridge.

Paek S. W. (2012) A multi-functional paintball cloud for asteroid deflection. 63rd International Astronautical Congress, Naples, Italy, IAC-12-A3.4.13.

Popova O. P., Jenniskens P., Emel'yanenko V., et al. (2013) Chelyabinsk airburst, damage assessment, meteorite recovery, and characterization. Science, 342, 1069-1073, DOI: 10.1126/ science. 1242642

Rozitis B., MacLennan E., and Emery J. P. (2014) Cohesive forces prevent the rotational breakup of rubble-pile asteroid (29075) 1950 DA. Nature, 512, 174-176, DOI: 10.1038/ nature13632.

Sánchez P. and Scheeres D. J. (2014) The strength of regolith and rubble pile asteroids. Meteoritics \& Planet. Sci., 49, 788-811, DOI: 10.1111/maps. 12293.

Shapiro I. I., Vilas F., A'Hearn M., et al. (2010) Defending Planet Earth: Near-Earth Object Surveys and Hazard Mitigation Strategies. National Academies, Washington DC.

Silber E. A., ReVelle D. O., Brown P. G., and Edwards W. N. (2009) An estimate of the terrestrial influx of large meteoroids from infrasonic measurements. J. Geophys. Res., 114, E08006, DOI: 10.1029/2009JE003334.

Stokes G. H., Yeomans D. K., Bottke W. F., et al. (2003) Study to Determine the Feasibility of Extending the Search for Near-Earth
Objects to Smaller Limiting Diameters. Report of the Near-Earth Object Science Definition Team at the Request of NASA's Office of Space Science, Solar System Exploration Division, available online at http://neo.jpl.nasa.gov/neo/neoreport030825.pdf.

Tancredi G., Ishitsuka J., Schultz P. H., Harris R. S., Brown P., Revelle D. O., Antier K., Le Pichon A., Rosales D., Vidal E., Varela M. E., Sánchez L., Benavente S., Bojorquez J., Cabezas D., and Dalmau A. (2009) A meteorite crater on Earth formed on September 15, 2007: The Carancas hypervelocity impact. Meteoritics \& Planet. Sci., 44, 1967-1984, DOI: 10.1111/j.1945-5100.2009.tb02006.x.

Tonry J. L. (2011) An early warning system for asteroid impact. Publ. Astron. Soc. Pac., 123, 58-73, DOI: 10.1086/657997.

Toon O. B., Zahnle K., Morrison D., Turco R. P., and Covey C. (1997) Environmental perturbations caused by the impacts of asteroids and comets. Rev. Geophys., 35, 41-78, DOI: 10.1029/96RG03038.

Trilling D. E., Mueller M., Hora J. L., Harris A. W., Bhattacharya B., Bottke W. F., Chesley S., Delbo M., Emery J. P., Fazio G., Mainzer A., Penprase B., Smith H. A., Spahr T. B., Stansberry J. A., and Thomas C. A. (2010) ExploreNEOs. I. Description and first results from the warm Spitzer near-Earth object survey. Astron. J., 140 , 770-784, DOI: 10.1088/0004-6256/140/3/770.

Tsuda Y., Yoshikawa M., Abe M., Minamino H., and Nakazawa S. (2013) System design of the Hayabusa 2 - Asteroid sample return mission to 1999 JU3. Acta Astronaut., 91, 356-362, DOI: 10.1016/j. actaastro.2013.06.028

Werner M. W., Roellig T. L., Low F. J., et al. (2004) The Spitzer Space Telescope mission. Astrophys. J. Suppl., 154, 1-9, DOI: $10.1086 / 422992$

Wolters S. D., Ball A. J., Wells N., Saunders C., and McBride N. (2011) Measurement requirements for a near-Earth asteroid impact mitigation demonstration mission. Planet. Space Sci., 59, 1506-1515, DOI: $10.1016 /$ j.pss.2011.06.015.

Wright E. L., Eisenhardt P. R. M., Mainzer A. K., et al. (2010) The Wide-field Infrared Survey Explorer (WISE): Mission description and initial on-orbit performance. Astron. J., 140, 1868-1881, DOI: 10.1088/0004-6256/140/6/1868.

Wünnemann K., Weiss R., and Hoffmann K. (2007) Characteristics of oceanic impact-induced large water waves - Re-evaluation of the tsunami hazard. Meteoritics \& Planet. Sci., 42, 1893-1903, DOI: 10.1111/j.1945-5100.2007.tb00548.x.

Yeomans D. K., Bhaskaran S., Broschart S. B., Chesley S. R., Chodas P. W., and Sweetser T. H. (2009) Deflecting a hazardous near-Earth object. 1st IAA Planetary Defense Conference: Protecting Earth from Asteroids, Granada, Spain, available online at http://neo.jpl. nasa.gov/neo/pdc paper.html. 